\title{
CHROMOSOME PAIRING AND SYNAPTONEMAL COMPLEX FORMATION IN HEXAPLOID WHEAT, MONOSOMIC FOR CHROMOSOME 5B
}

\author{
by
}

\author{
PREBEN BACH HOLM
}

\author{
Department of Physiology, Carlsberg Laboratory, \\ Gamle Carlsberg Vej 10, DK-2500 Copenhagen Valby
}

Keywords: Homoeologues, allopolyploidy, crossing over, Triticum aestivum

\begin{abstract}
Chromosome pairing and synaptonemal complex formation have been analyzed at the ultrastructural level in spread microsporocyte nuclei of allohexaploid wheat, monosomic for chromosome $5 B$. The analysis comprised partial tracing of lateral components and synaptonemal complexes of nuclei at the leptotene-zygotene transition and more complete tracing of $\mathbf{3 0}$ nuclei ranging in stage from early zygotene to early diplotene. In addition, four early-mid zygotene nuclei of euploid wheat were traced. At the leptotene to zygotene transition the individual lateral component aligned with several other lateral components over short distances along its length. Pairing partner exhanges were frequent at zygotene and the number of lateral components involved in multiple associations exceeded that observed in euploid wheat by a factor of two. At pachytene only $90 \%$ of the complement had synapsed and most multiple associations persisted during pachytene into diplotene. It is assumed that some of the multiple associations represent pentavalents consisting of the five homoeologues of group 5. As monosomic 5B virtually never possesses multivalents at metaphase $\mathrm{I}$, it is concluded that the presence of just one copy of chromosome $5 B$ is sufficient to suppress crossing over between homoeologues.
\end{abstract}

\section{INTRODUCTION}

The present paper is addressed to an investigation of chromosome pairing and synaptonemal complex. formation in allohexaploid wheat monosomic for chromosome 5B. It is a part of an ultrastructural investigation of meiosis in wheat attempting to define the effect of chromosome $5 \mathrm{~B}$ on synapsis and chiasma formation. The study involves analyses of allohexaploid wheat nullisomic for chromosome $5 \mathrm{~B}(4)$, euploid wheat $(2,3,7)$, allohexaploid wheat where chromosome $5 \mathrm{~B}$ has been replaced by one, two or three isochromosomes for the long arm of chromosome 5B $(5,15)$, trihaploid wheat with and without $5 \mathrm{~B}(11)$ and wheat/rye hybrids with chromosome 5B, without $5 \mathrm{~B}$ or carrying an isochromosome for the long arm of chromosome $5 \mathrm{~B}(12)$. The significance of the combined results of these ultrastructural investigations for chromosome pairing in allopolyploids and especially in wheat is discussed in a separate paper (6).

\section{MATERIALS AND METHODS}

Seeds of allohexaploid wheat, $T$. aestivum $\mathrm{cv}$. Chinese Spring monosomic for chromosome 5B were kindly provided by Dr. TERRY MILLER, Plant Breeding Institute, Cambridge. One set of plants was grown at $20{ }^{\circ} \mathrm{C}$ in a heated glass house. The light period was in the spring and autumn prolonged by artificial illumination and 

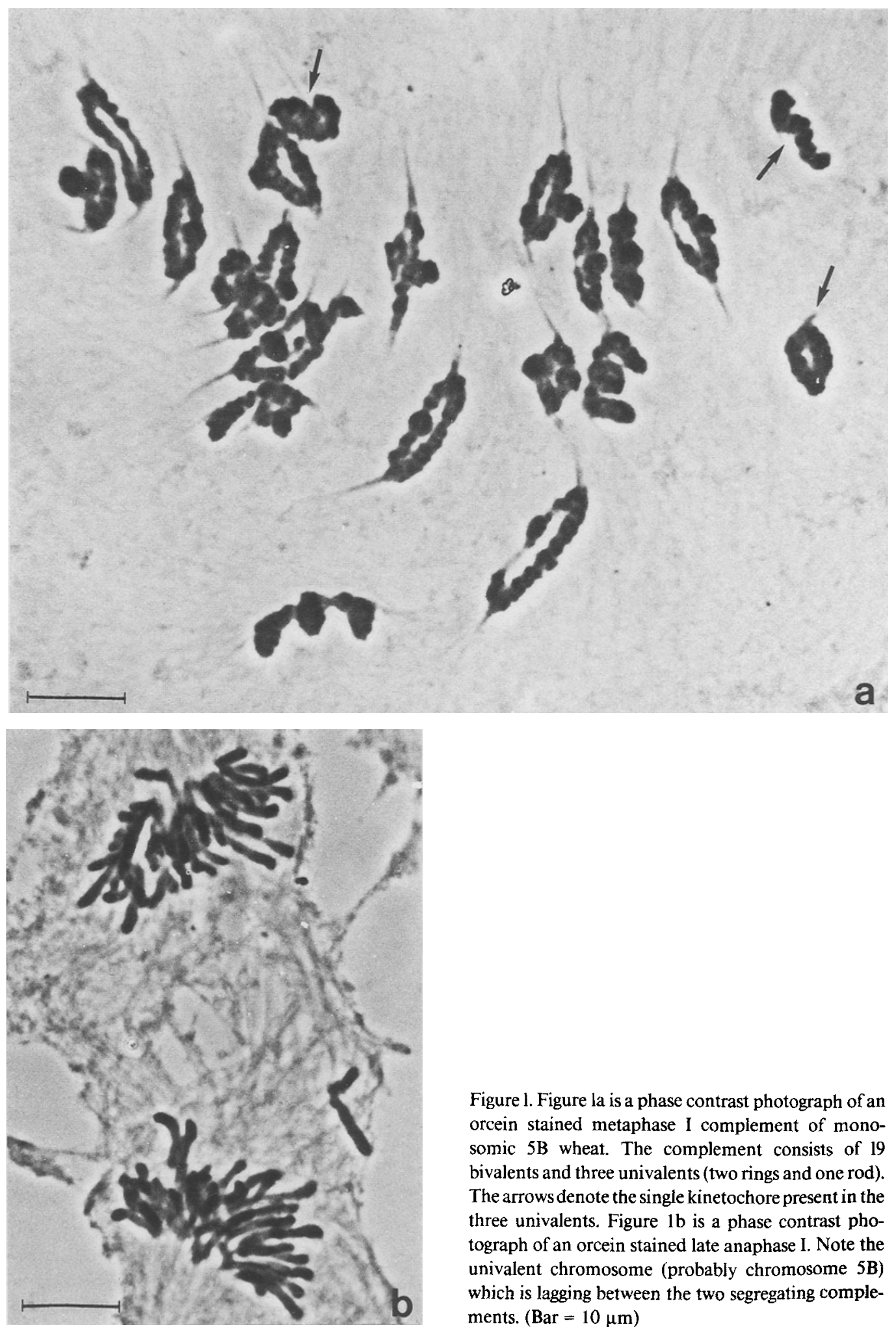

Figure l. Figure la is a phase contrast photograph of an orcein stained metaphase I compiement of monosomic 5B wheat. The complement consists of 19 bivalents and three univalents (two rings and one rod). The arrows denote the single kinetochore present in the three univalents. Figure $1 \mathrm{~b}$ is a phase contrast photograph of an orcein stained late anaphase I. Note the univalent chromosome (probably chromosome 5B) which is lagging between the two segregating complements. $(\mathrm{Bar}=10 \mu \mathrm{m})$ 


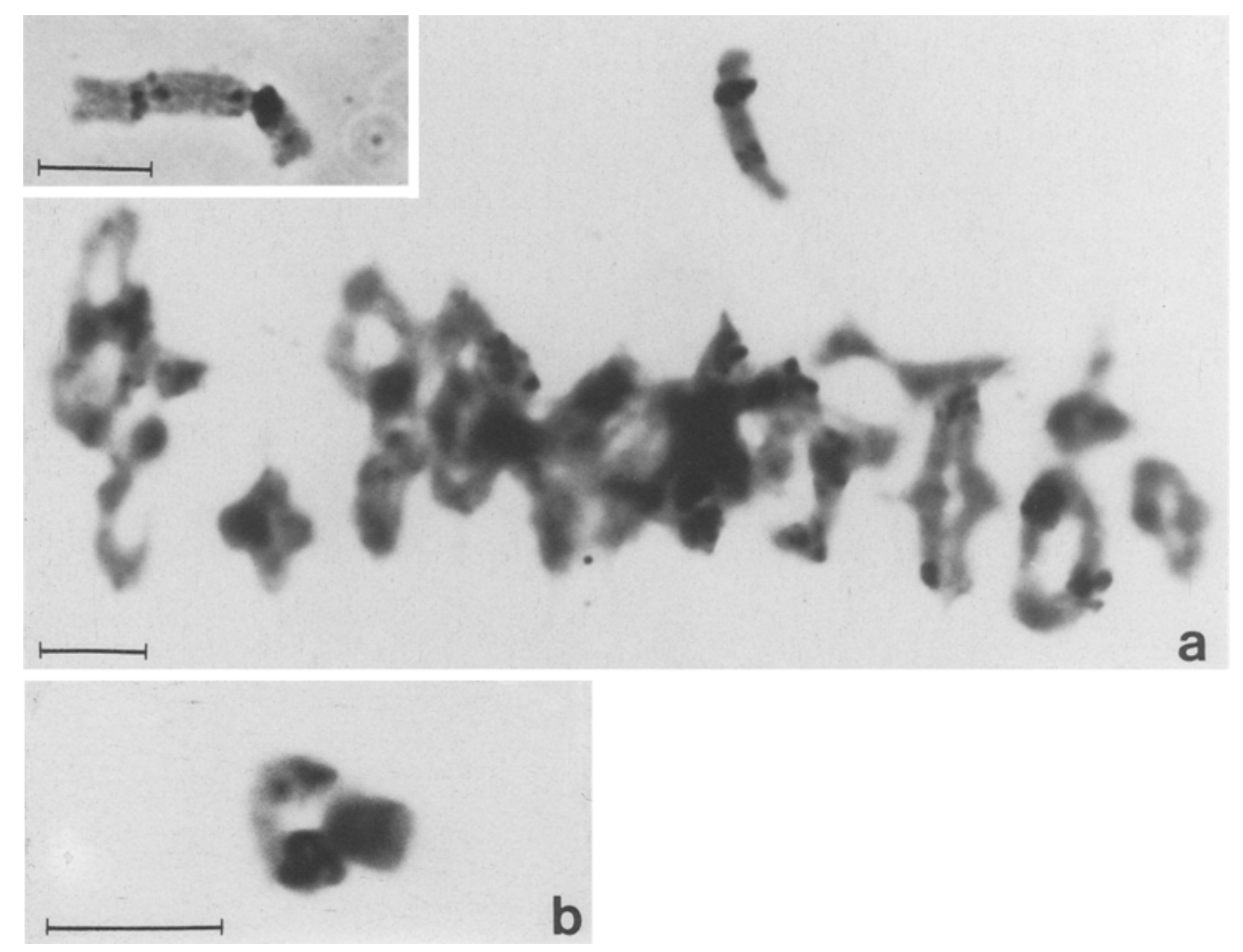

Figure 2. Photographs of $\mathrm{N}$-banded chromosomes and bivalents of monosomic $5 \mathrm{~B}$ wheat. Figure 2a shows an $\mathrm{N}$-banded metaphase I complement. Note the univalent chromosome $5 \mathrm{~B}$ lying outside the metaphase plane with its prominent $\mathrm{N}$-band proximal in the short arm and a double band in the middle of the long arm. The insert shows an $\mathrm{N}$-banded chromosome $5 \mathrm{~B}$ from a root tip metaphase. Figure $2 \mathrm{~b}$ illustrates the occasional ring formation of chromosome 5B due to an association between the ends of the long and the short arms. (Bar $=5 \mu \mathrm{m})$

during the wintertime the plants were grown under continuous light. Other plants were grown in a growth cabinet at $15^{\circ} \mathrm{C}$ during daytime and $10^{\circ} \mathrm{C}$ at night with a light period of 16 hours and 8 hours darkness. The plants were transferred to a Weiss growth cabinet before reaching the tillering stage and grown at $20^{\circ} \mathrm{C} /$ $15^{\circ} \mathrm{C}$, using the same light period as described above. The data presented here are based on analyses of two glass house and two growth cabinet grown plants, but as no differences were observed between the four plants, the results were pooled.

The genomic constitution of the plants was analyzed by light microscopy using conventional staining or $\mathrm{N}$-banding of the chromosome complements of root tips and metaphase I complements. Fresh root tips about $1 \mathrm{~cm}$ long were excised and placed at $0{ }^{\circ} \mathrm{C}$ in ice water for 22 hours, whereafter they were fixed in a $3: 1 \mathrm{mix}-$ ture of absolute ethanol and glacial acetic acid. After fixation the root tips were washed in distilled water and treated with cellulase/pectinase as described by WANG and HU (13). After refixation in the $3: 1$ mixture, the root tips were squashed in orcein or carbol fuchsin and the preparations made permanent by freezing on dry ice followed by dehydration and mounting in Eukitt. For $\mathrm{N}$-banding the squashing was performed in $45 \%$ acetic acid, whereafter the slides were processed as described by ENDO and GILL (1). Anthers were processed similarly, 


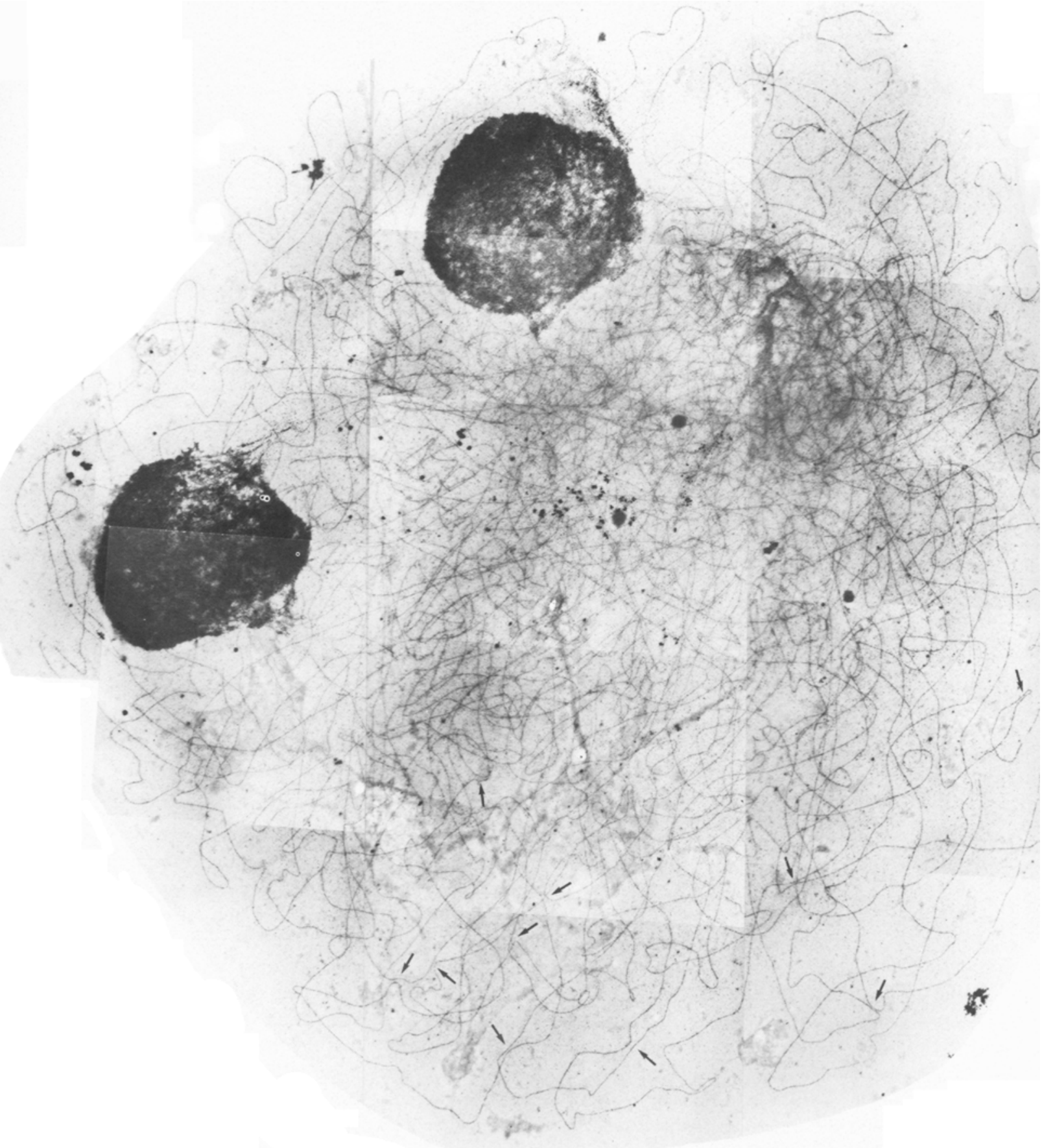



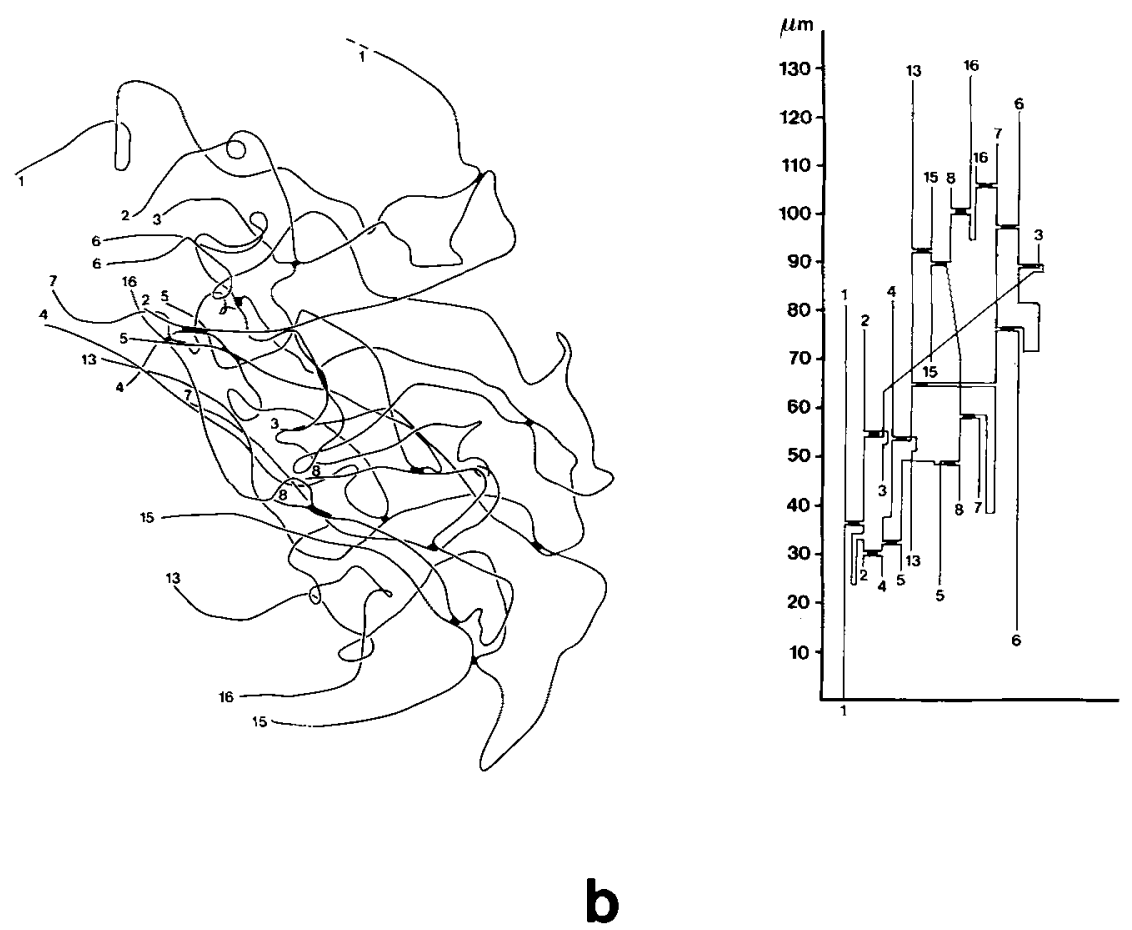

C

Figure 3. Figure $3 a$ is a montage of micrographs illustrating the fine structure of a spread nucleus of monosomic $5 \mathrm{~B}$ wheat at the leptotene to zygotene transition. The telomeres are aggregated in the upper half of the nucleus and a few are paired with a synaptonemal complex. The arrows denote a few of the regions where segments of lateral components are closely aligned with other lateral components. Figures $3 \mathrm{~b}$ and $3 \mathrm{c}$ are a tracing and a diagrammatic presentation of some of the lateral components in Figure 3a. Regions of close alignment are shown in black. (Bar $=10 \mu \mathrm{m})$

omitting the enzyme treatment step.

Spreading of the microsporocytes as well as all the procedures for silver staining of the synaptonemal complexes and unpaired lateral components, electron microscopy, tracing and measuring were performed as described previously (3). In some of the preparations the postfixation step in glutaraldehyde and the washing in Ficoll were omitted.

\section{RESULTS}

\subsection{Light microscopy}

At metaphase I there were in almost all cells 20 bivalents and a single univalent chromosome, usually located outside the metaphase plane and lagging on the spindle at anaphase I (Figure 1). The bivalents were nearly always ring bivalents and the number of chiasmata similar to that found in euploid wheat. N-banding revealed that the univalent possessed the morphology and banding patterns characteristic for chromosome $5 \mathrm{~B}$, which is a large submetacentric chromosome with a prominent $\mathrm{N}$-band proximal in the short arm, a faint band distal in the short arm, a faint proximal band in the long arm and a more distinct medial double band in the long arm (Figure 2) (1). In a few cases, however, additional univalents were present, often forming small univalent rings containing a single kinetochore (Figure 1a). Chromosome $5 \mathrm{~B}$ was also in some cases present as a ring univalent (Figure 2b).

Selfing of monosomic 5B plants results, as in other hexaploid wheat monosomics, in progeny where about $75 \%$ of the plants are monosomic. The high frequency of transmission of the univa- 

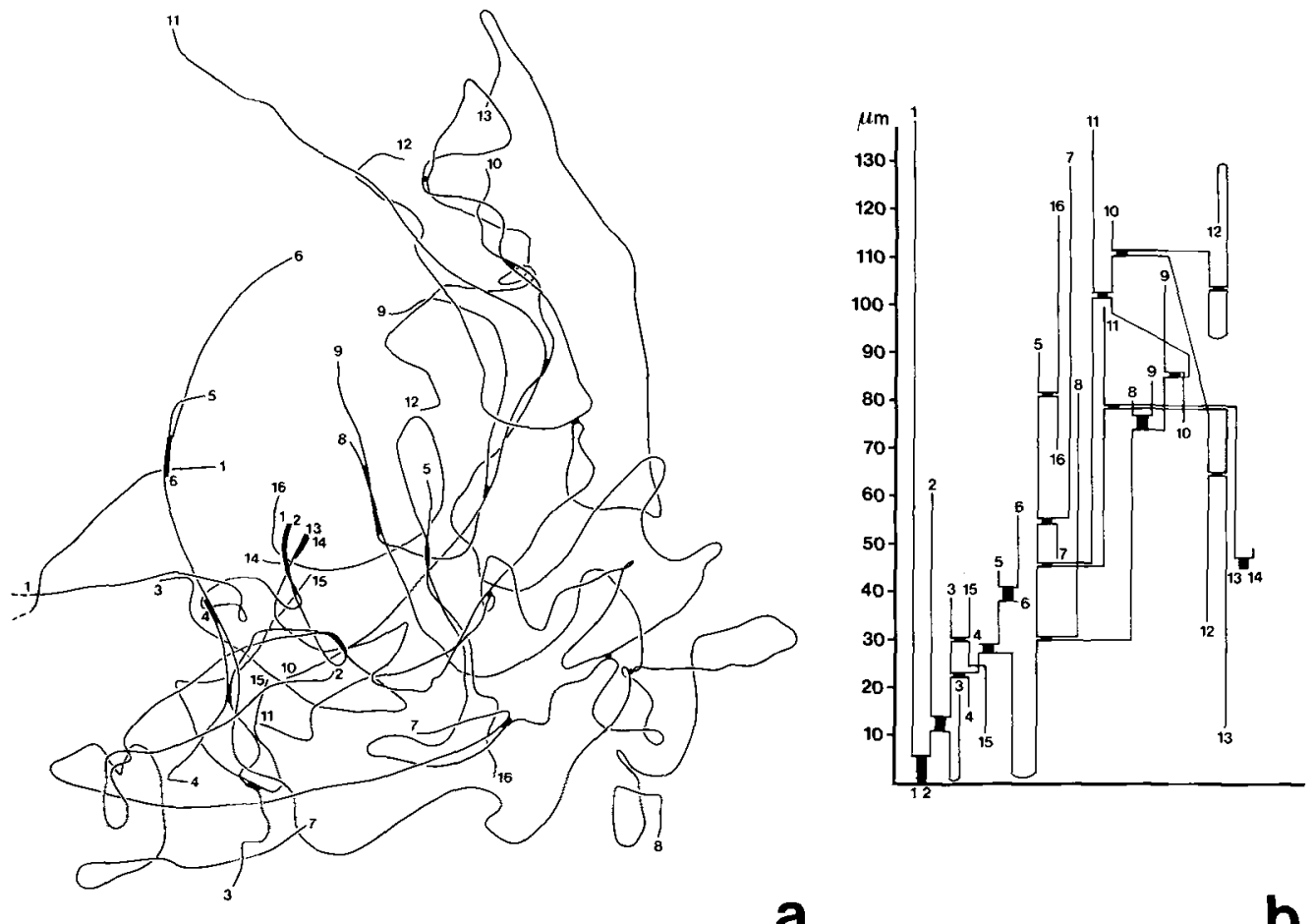

Figure 4. A tracing (Figure 4a) and a diagrammatic presentation (Figure 4b) of the alignment between lateral components in a spread nucleus of euploid wheat at the leptotene to zygotene transition. Regions of close alignment are shown in black. The tracings are from the nucleus shown as Figure 1 in (3).

lent chromosome results from the combined effects of a strong selection against pollen carrying only 20 chromosomes and a preferential segregation of the univalent on the female side where only $25 \%$ of the egg cells receive the univalent. In one percent of the cases does selfing of monosomic $5 \mathrm{~B}$ wheat result in plants nullisomic for $5 \mathrm{~B}$ while the remaining plants (about 25\%) are euploid (10). Generation of isochromosomes for the long arm of chromosome $5 \mathrm{~B}$ by fission of the centromere of chromosome 5B at anaphase I followed by fusion between the centromere ends of the two sister chromatids is also frequent, as such isochromosomes are regularly found in the progeny of monosomic $5 \mathrm{~B}$ plants (5). As chromosome $5 \mathrm{~B}$ as well as the isochromosome have an easily recognizable $\mathrm{N}$-banding pattern, $\mathrm{N}$-banding of root tip metaphases or metaphase I provides a reliable method for assessing the genomic constitu- tion of the progeny of selfed monosomic 5B wheat.

\subsection{Electron microscopy}

\subsubsection{General aspects}

As described in detail previously (3) spreading of nuclei of allohexaploid wheat and tracing of the silver stained lateral components meet with several difficulties when the aim is to trace the 42 lateral components with a combined length of 4-5 $\mathrm{mm}$ at zygotene. The major problem encountered is the presence of broken lateral components and more rarely broken synaptonemal complexes. Breakage appears in some cases to result from stretching of lateral components during swelling and/or air drying and flattening of the nuclei, but can more frequently be attributed to breakage of lateral components in resolving interlockings. Tracing of overlapping 


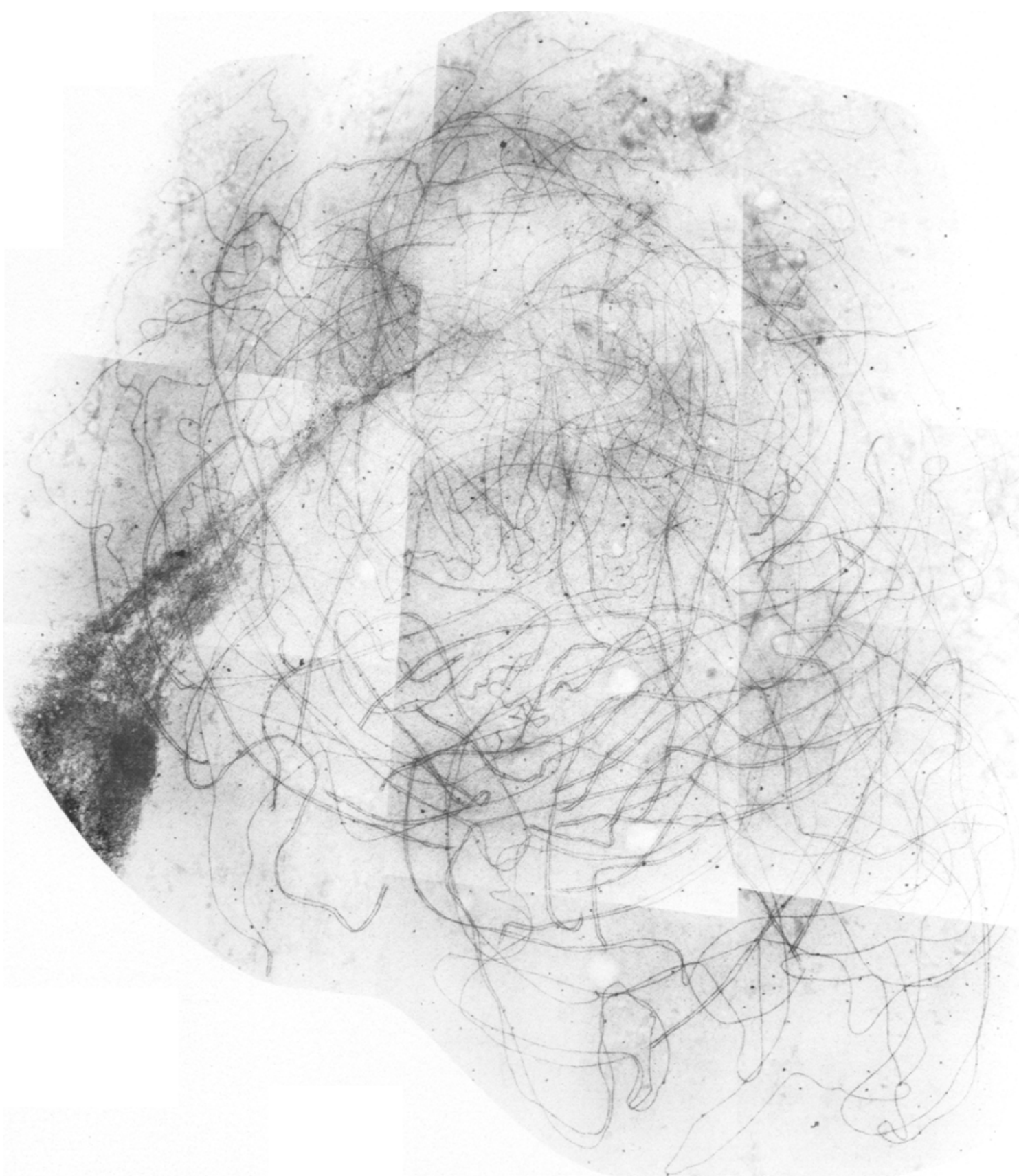

Figure 5. A montage of micrographs of an early zygotene nucleus of monosomic 5B wheat (Z8, 38\% pairing). The telomeres of the lateral components are aggregated on one side of the spread nucleus. $(\operatorname{Bar}=10 \mu \mathrm{m})$ 


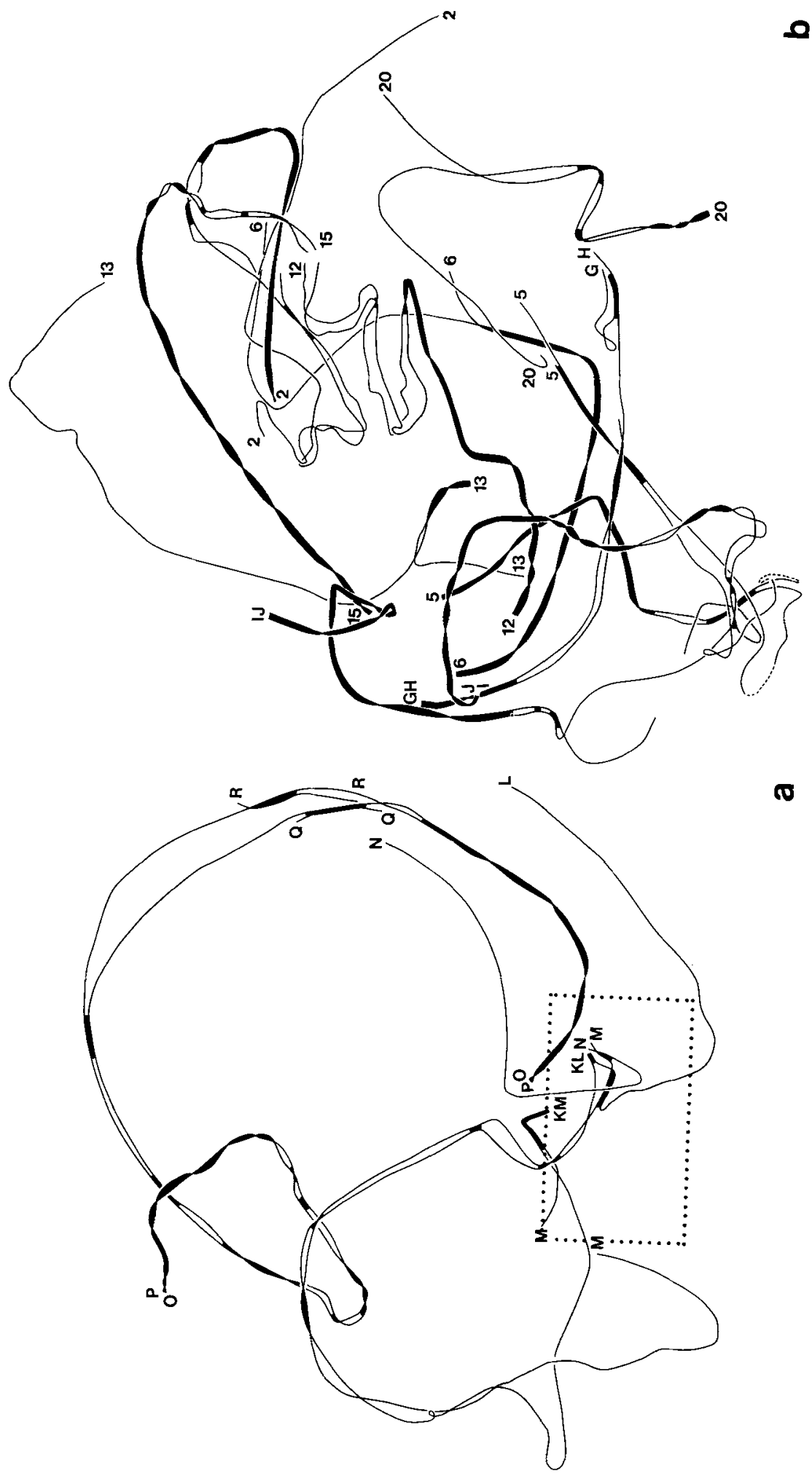




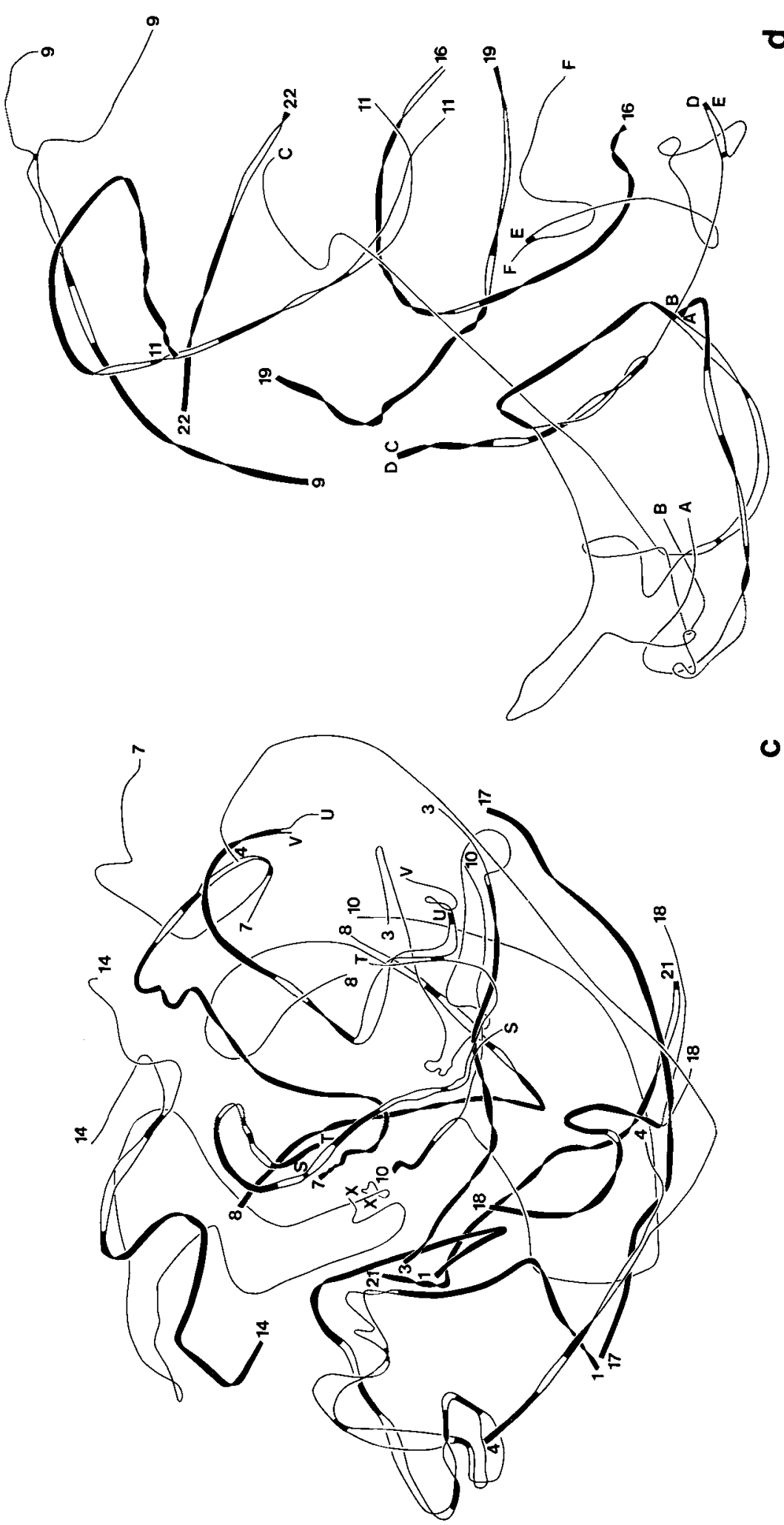

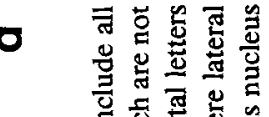

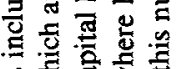

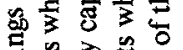

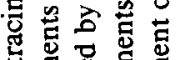
胥范

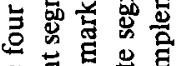
苛焉 F吉芯总 की

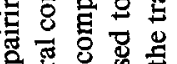

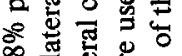
赵焉莺

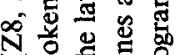
㐘的总

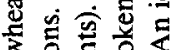
总至员 为怘焉

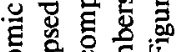
品范焉 公要要 론형 틍응

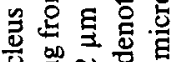

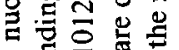

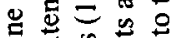

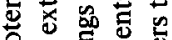

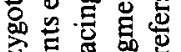
సิ

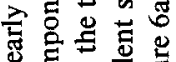
동.

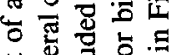

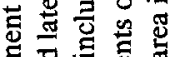

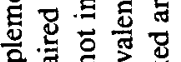
를응 可守包 × 흘

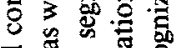
范芯焉 总言总。

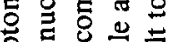
产㤩总总 会艺焉 幽

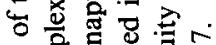

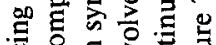

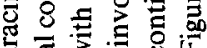

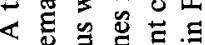
๑

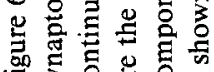




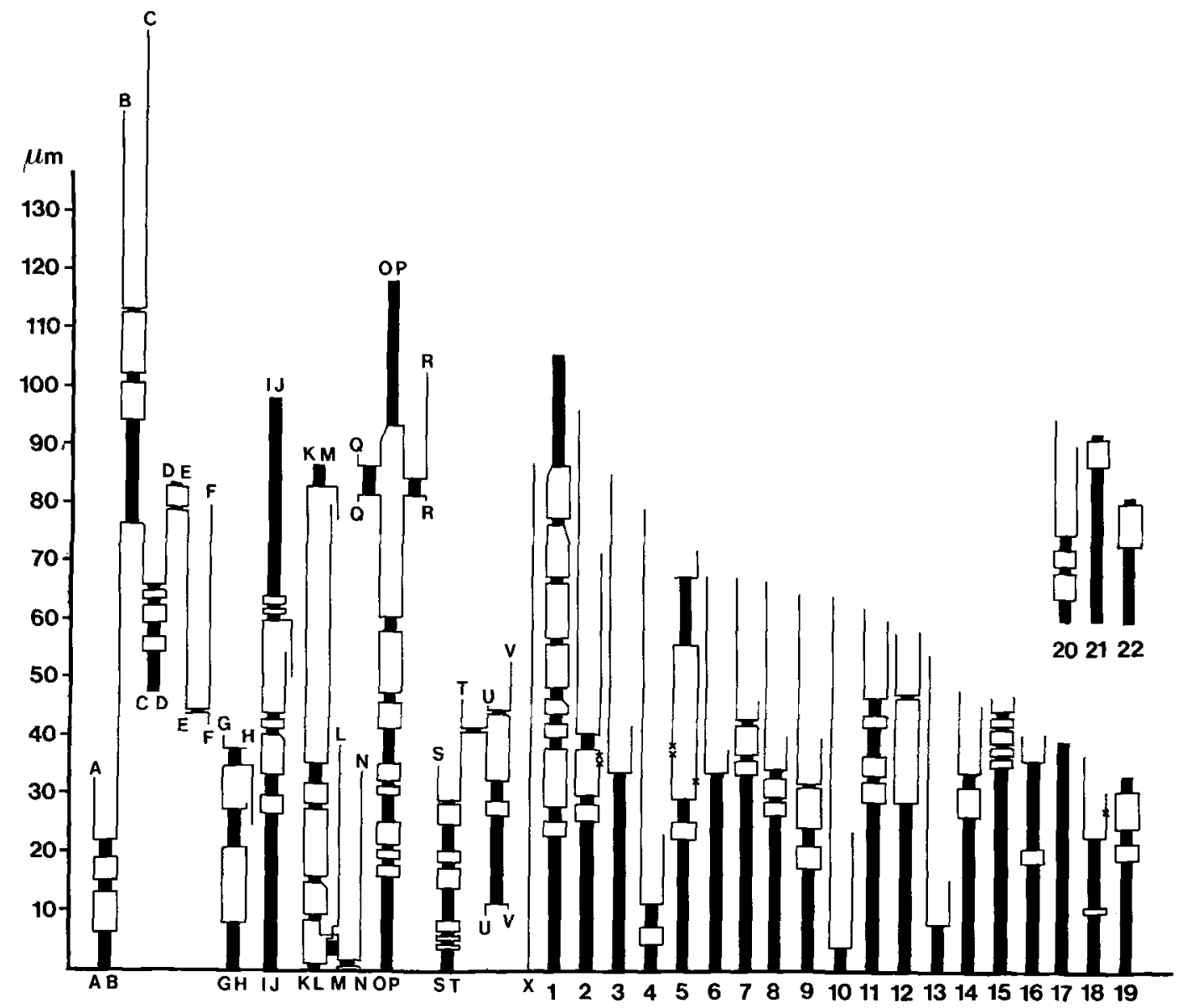

Figure 7. An idiogram of the traced complement of an early zygotene nucleus of monosomic 5B wheat (Z8, 38\% pairing). Unpaired segments of lateral components which, due to breakage, are not continuous with synaptonemal complexes have been excluded ( $1012 \mu \mathrm{m}$ of lateral component, $22 \%$ of the total complement length). Lateral components or lateral component segments in multiple associations are shown first and are referred to by capital letters while partially paired bivalents or bivalent segments are denoted by numbers and arranged after decreasing size. The actual lengths of the synaptonemal complexes and the lateral components are represented by the vertical lines. Breaks in lateral components are indicated by crosses. A montage of micrographs of this nucleus is shown in Figure 5 and tracings of the complement in Figure 6.

and intertwined unpaired lateral components is often difficult, usually hindering complete tracings of early zygotene nuclei. For these reasons a large fraction of the spread nuclei, in particular at the early stages was impossible to trace in sufficient detail. Spread nuclei containing a large, dense nucleolus in the middle of the flattened nucleus were also discarded, as the nucleolar structure then covers several lateral components and synaptonemal complex seg- ments. Finally, as described for euploid wheat (3) the lateral component complement can be stretched to varying degree in different nuclei, which probably accounts for the substantial variation in complement length among nuclei at the same stage. Nuclei showing extensive stretching of the lateral components were not analyzed.

Hence, the analysis is based on a selected sample of spread nuclei. It is considered unlike- 


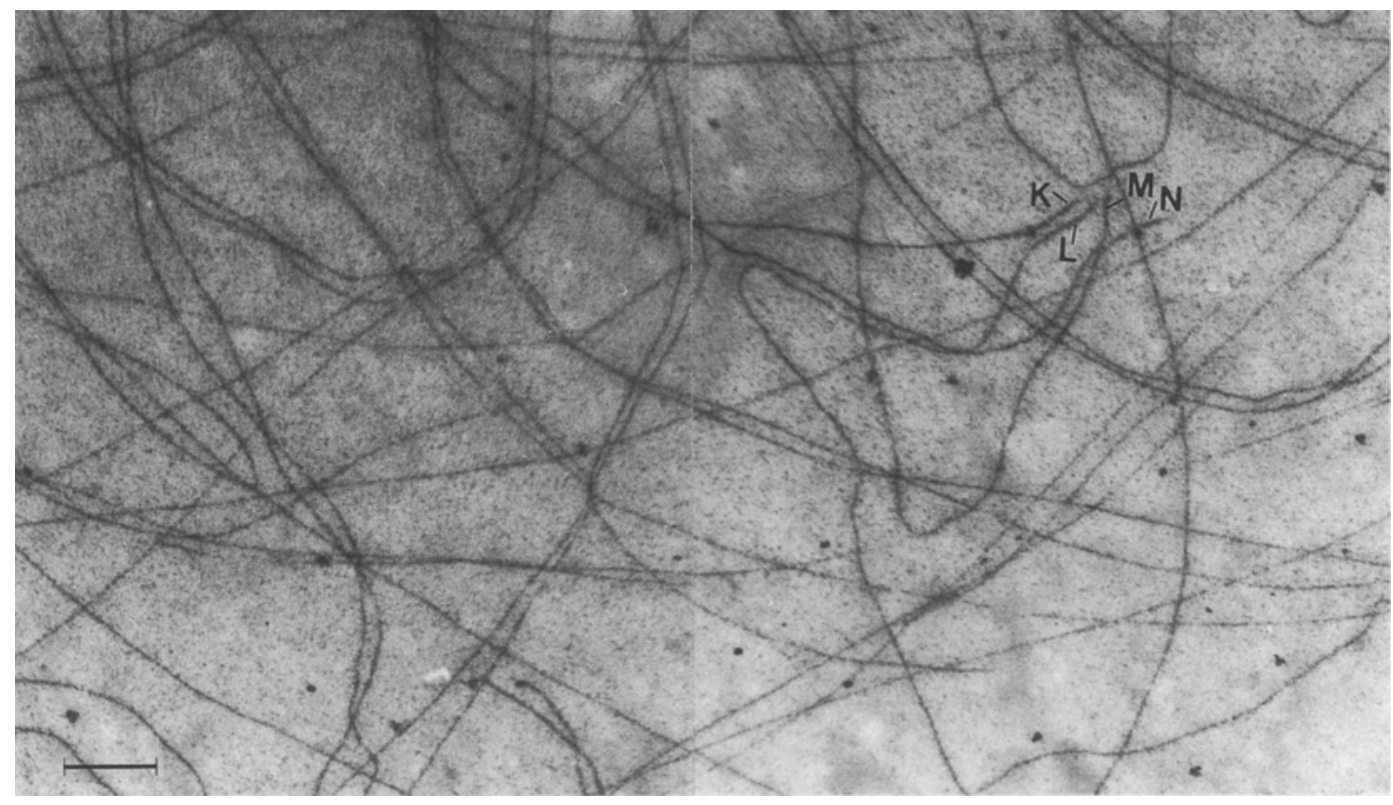

Figure 8. Micrographs of part of an early zygotene nucleus (Z8, 38\% pairing) of monosomic 5B wheat. The lateral components denoted $\mathrm{K}, \mathrm{L}, \mathrm{M}$ and $\mathrm{N}$ exchange pairing partners close to the telomeres. Tracings of the entire complement of this nucleus is shown in Figure 6 and an idiogram in Figure 7. $(\mathrm{Bar}=1 \mu \mathrm{m})$

ly, however, that these nuclei with respect to pairing and synaptonemal complex formation deviate significantly from the untraceable nuclei as partial tracings of such nuclei reveal the same pairing characteristics as those observed in the more easily analyzed nuclei.

The morphological characteristics of the euploid nuclei at the different stages of meiotic prophase and the pairing kinetics of their chromosomes have been described in detail previously (3). Hence, the present study of monosomic 5B wheat will primarily be addressed to a description of differences in the structural characteristics and the pairing kinetics of euploid wheat and monosomic 5B wheat.

\subsubsection{Early-mid zygotene}

As in euploid wheat the lateral components first appear as short discrete segments in the nuclei, whereafter lateral component continuity is established. At the same time a congression of the telomeres is apparent, nucleolar fusion occurs and synapsis commences. Throughout this interval there is no indication of a large scale two-by-two alignment of lateral components or alignment between groups of lateral components. The fine structure of a spread nucleus at the leptotene to zygotene transition is shown in Figure 3a. Pairing and synaptonemal complex formation have occurred in a few segments, primarily at or close to the telomeres. In several interstitial regions alignment is in progress. It cannot be excluded that synaptonemal complexes are assembled in such regions since the central region usually is not stained in the spreads, but in most cases the distance between two aligned lateral component segments is somewhat greater than the diameter of the central region of the synaptonemal complex. The juxtaposition of these lateral components is probably caused by alignment and not by synaptonemal complex formation.

A complete tracing of the complement is not possible at the leptotene to zygotene transition stage. Partial tracings reveal, however, that the alignment is not restricted to pairs of lateral components. Rather, the individual lateral com- 
Table I. Degree of pairing, lateral component (LC) length of one complement $(1 / 2 \mathrm{LC}$ length) and the number of lateral components or lateral component fragments involved in multiple associations in three early-mid zygotene nuclei of wheat microsporocytes monosomic for chromosome 5B. The number of multiple associations in parenthesis are switch back associations of type II (see section 3.2.2 for explanation).

\begin{tabular}{llll}
\hline $\begin{array}{l}\text { Nucleus } \\
\text { number }\end{array}$ & \% pairing & $\begin{array}{l}1 / 2 \text { LC length } \\
\mu \mathrm{m}\end{array}$ & $\begin{array}{l}\text { Number of LC's in } \\
\text { multiple associations }\end{array}$ \\
\hline 8 & 38 & 2352 & $25(8)$ \\
9 & 39 & 2445 & $13(3)$ \\
5 & 50 & 2228 & $7(0)$ \\
\hline Mean & 42 & 2342 & $15(4)$ \\
\hline
\end{tabular}

Table II. Degree of pairing, lateral component (LC) length of one complement $(1 / 2$ LC length) and the number of lateral components or lateral component fragments involved in multiple associations in five early-mid zygotene nuclei in microsporocytes of euploid wheat. The number of multiple associations in parenthesis are switch back associations of type II (see section 3.2.2 for explanation). s.d., standard deviation.

\begin{tabular}{llllc}
\hline $\begin{array}{l}\text { Nucleus } \\
\text { number }\end{array}$ & \% pairing & $\begin{array}{l}1 / 2 \text { LC length } \\
\mu \mathrm{m}\end{array}$ & \multicolumn{2}{c}{$\begin{array}{l}\text { Number of } L C \text { 's in } \\
\text { multiple associations }\end{array}$} \\
\hline 1 & 27 & 1971 & 10 & $(10)$ \\
2 & 38 & 1547 & 4 & $(4)$ \\
5 & 40 & 2362 & 4 & $(4)$ \\
3 & 43 & 1762 & 12 & $(6)$ \\
7 & 45 & 2275 & 10 & $(10)$ \\
\hline Mean \pm s.d. & $39 \pm 7$ & $1984 \pm 342$ & $8 \pm 4$ & $(7 \pm 3)$ \\
\hline
\end{tabular}

ponent aligns with several other lateral components over short distances along its length whereby virtual "networks" of aligned lateral components form (Figures $3 \mathrm{~b}$ and $3 \mathrm{c}$ ). In some cases the lateral components fold back and align with themselves.

A similar alignment between several different lateral components is also apparent in euploid wheat. Figure $4 a$ is a tracing of a part of the leptotene-zygotene nucleus shown in Figure 1 in the study of euploid wheat (3), illustrating that just within a small fraction of this nucleus at least 16 lateral component segments are interconnected due to alignment with two or more partners in different regions (Figure $4 \mathrm{~b}$ ). It has not been attempted to determine if there are differences in the alignment between the euploid and the monosomic 5B genotypes.

As chromosome pairing and synaptonemal complex formation progress during early zygotene the "network" of multiply aligned lateral components is no longer apparent. Tracings of the synaptonemal complexes and unpaired lateral components of three nuclei with 38,39 and $50 \%$ pairing (Table I) revealed that as in euploid wheat synaptonemal complex formation proceeds primarily from the telomeres but also occurs interstitially (Figures 5, 6 and 7).

As is the case for euploid wheat, an identification of the individual wheat chromosomes in the

Figure 9. Idiograms of the synaptonemal complex complement of two early zygotene nuclei of euploid wheat (EZ1, $27 \%$ pairing, Figure $9 \mathrm{a}$; EZ2, 38\% pairing, Figure $9 \mathrm{~b}$ ). For explanation of symbols, see legend to Figure 7. 

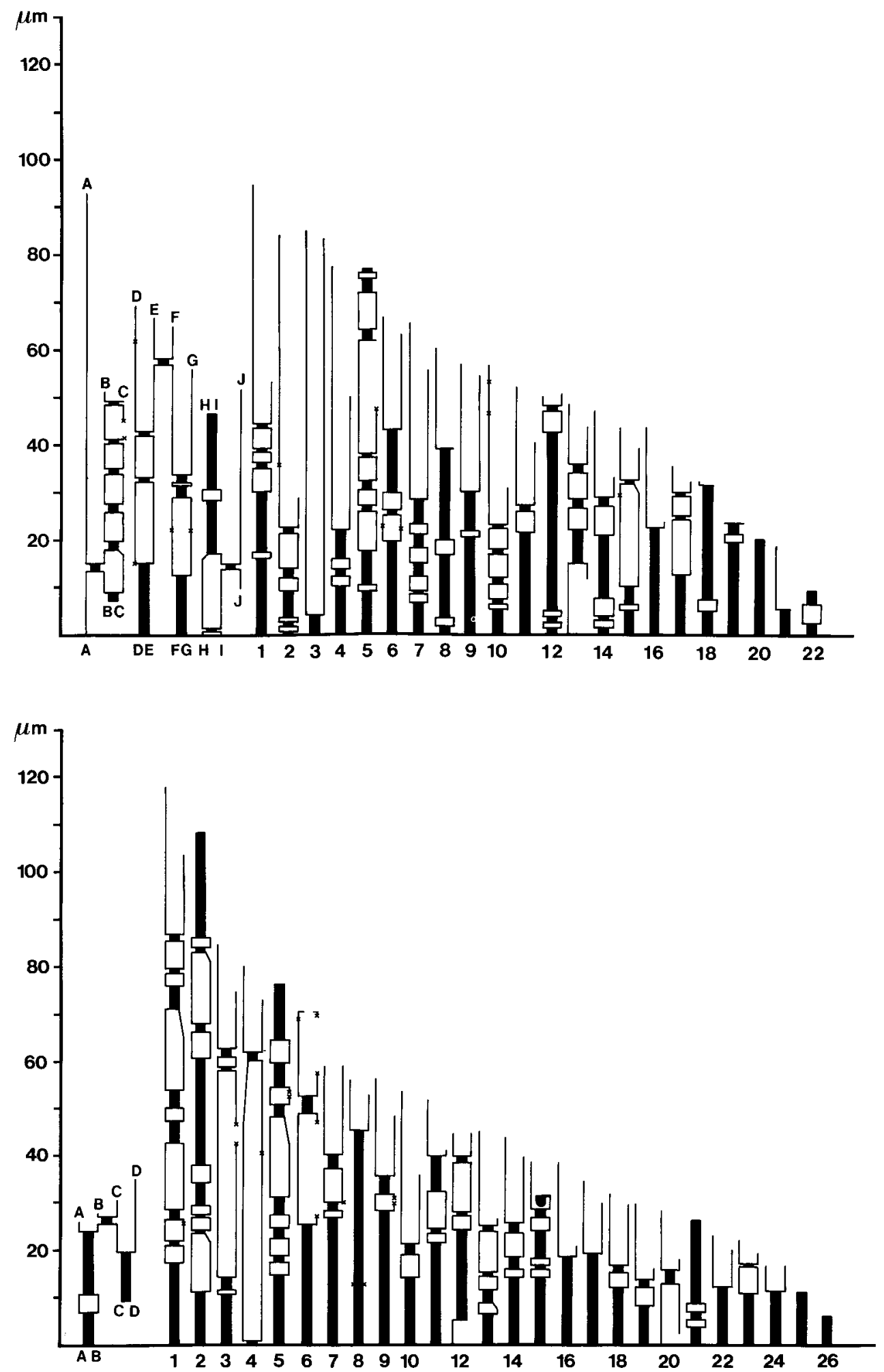
Table III. Degree of pairing, lateral component (LC) length of one complement $(1 / 2 \mathrm{LC}$ length) and the number of lateral components or lateral component fragments involved in multiple associations in six mid-late zygotene nuclei of wheat microsporocytes monosomic for chromosome 5B. The number of multiple associations in parenthesis are switch back associations of type II (see section 3.2.2 for explanation). s.d., standard deviation. The data for euploid wheat (3) are included for comparison.

\begin{tabular}{lllrl}
$\begin{array}{l}\text { Nucleus } \\
\text { number }\end{array}$ & \% pairing & $\begin{array}{l}1 / 2 \text { LC length } \\
\mu \mathrm{m}\end{array}$ & \multicolumn{2}{c}{$\begin{array}{l}\text { Number of LC's in } \\
\text { multiple associations }\end{array}$} \\
\hline 1 & 70 & 2035 & 17 & $(0)$ \\
7 & 73 & 1976 & 6 & $(0)$ \\
3 & 75 & 2137 & 12 & $(0)$ \\
6 & 81 & 1993 & 8 & $(0)$ \\
2 & 83 & 1739 & 10 & $(0)$ \\
4 & 91 & 1442 & 5 & $(0)$ \\
\hline Mean \pm s.d. & $79 \pm 8$ & $1887 \pm 254$ & $10 \pm$ & $(0)$ \\
\hline Euploid wheat & & & & \\
Mid zygotene & $62 \pm 7$ & $2040 \pm 164$ & $5 \pm 6$ & $(3 \pm 3)$ \\
Late zygotene & $93 \pm 1$ & $1806 \pm 160$ & $5 \pm 5$ & $(0)$ \\
\hline
\end{tabular}

spreads is not possible due to the lack of appropriate markers such as heterochromatic regions or centromeres. Furthermore, the breakage of the lateral components hampers a classification by length. In several nuclei two or three nucleolus organizing regions are evident, but since the lateral components of the nucleolus organizing chromosomes often are broken, presumably due to displacement of the nucleolus during the air drying of the nuclei, an identification of the nucleolus organizing chromosomes has not been attempted in this study.

Multiple associations of chromosomes resulting from pairing partner exchanges between lateral components are frequent in monosomic $5 \mathrm{~B}$ wheat at this early stage of pairing (Figures 6 , 7 and 8 and Table I). In a few cases the points of exchange are close to the telomere (lateral components $\mathrm{K}, \mathrm{L}, \mathrm{M}$ and $\mathrm{N}$ in Figures 6, 7 and 8), but the pairing partner exchanges have in general a more proximal location. A classification of the different associations into different types has not been attempted, the only exception being associations termed type II. These associations consist of two partially synapsed bivalents (or a bivalent and a univalent) in which a change of pairing partner occurs over a $0.5-1.0 \mu \mathrm{m}$ distance, whereafter the involved lateral components switch back to their original partner (e.g. the association between lateral components $S$ and $T$ with $U$ and $V$ in Figure 7). The remaining types of multiple associations include associations where the lateral components do not switch back to their original partner, associations involving more than four lateral components or associations of two lateral components which appear to be nonhomologous. The twoby-two associations between lateral components $G$ and $H, I$ and $J$, and $K$ and $M$ in Figure 7 are examples of the latter category of synapsis between chromosomes that probably are not homologous. The study of euploid wheat (3) thus revealed that in unpaired bivalent segments, where one lateral component is broken, the intact lateral component is almost invariably somewhat longer than the combined length of the two lateral components of the broken segment. In regions of interlocking the lateral components often appear to be stretched and the difference in length of the two lateral components may be attributed to stretching of the intact lateral component, while breakage of the other lateral component results in retainment of the original length or even some shortening due to contraction. Hence, associations of two lateral components, where a broken lateral component in an unpaired segment is longer than the intact one as is the case for the lateral component 


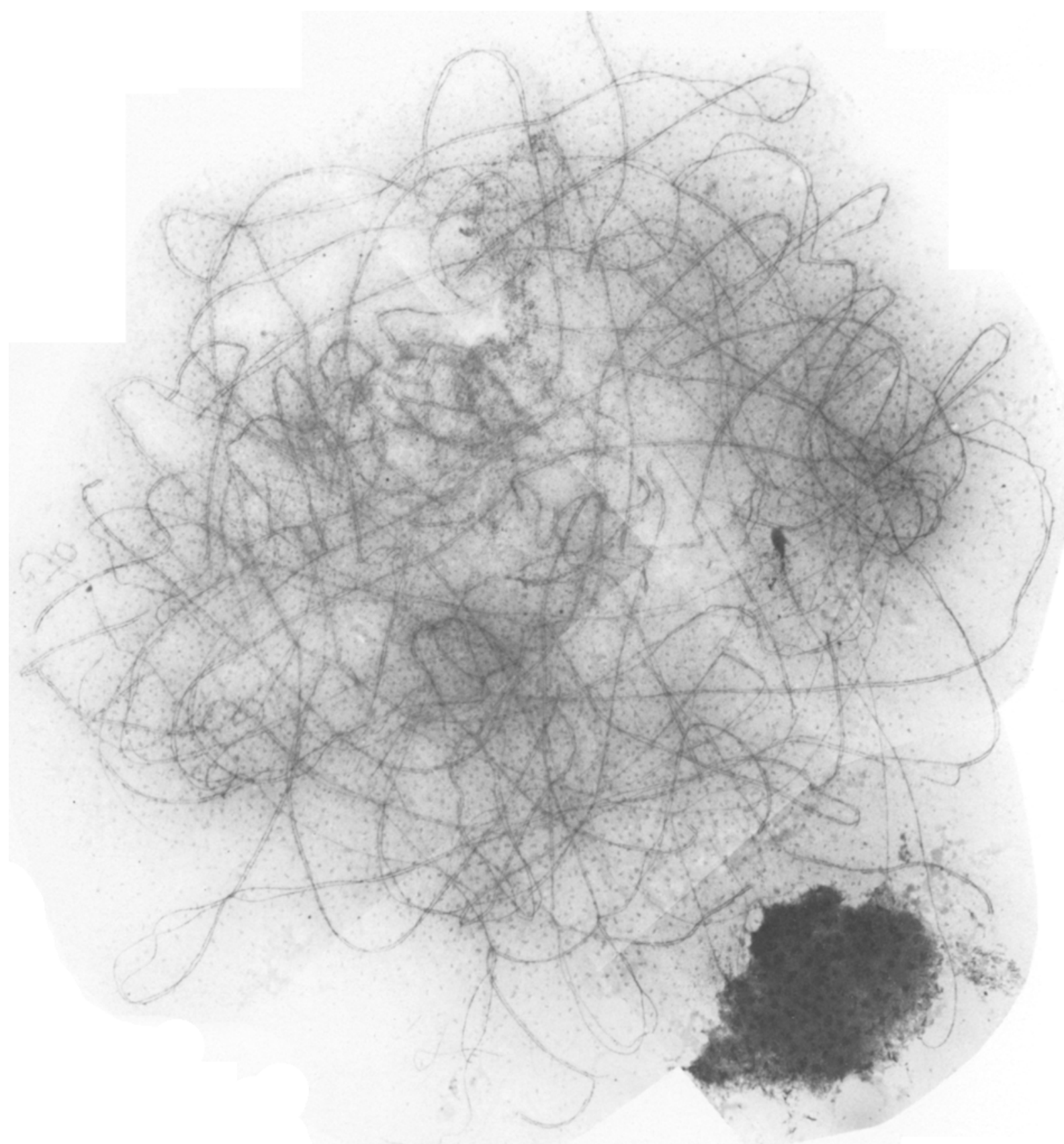

Figure 10. A montage of micrographs illustrating the fine structure of a mid-late zygotene nucleus of monosomic $5 \mathrm{~B}$ wheat $(\mathrm{Z3}, 75 \%$ pairing $)$. $(\mathrm{Bar}=10 \mu \mathrm{m})$ 


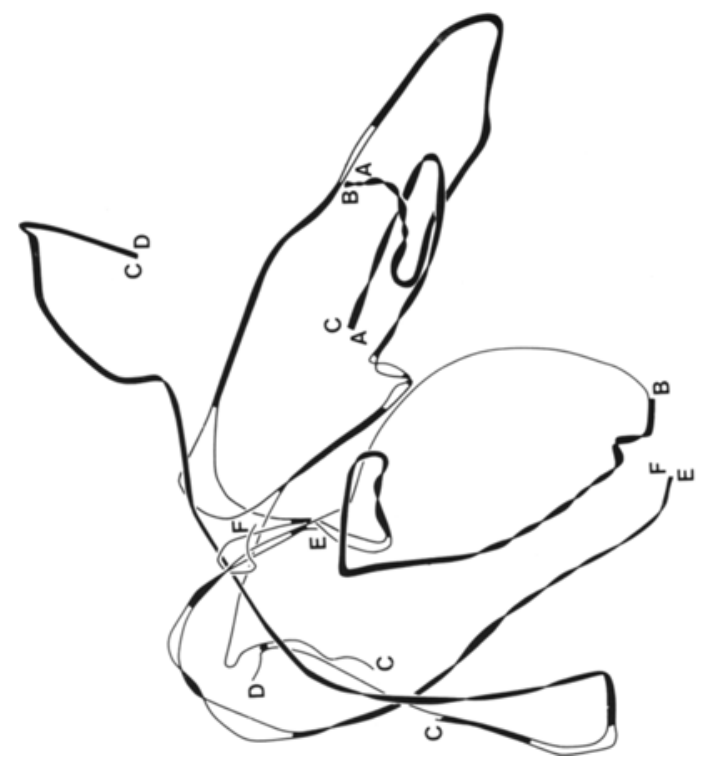

오

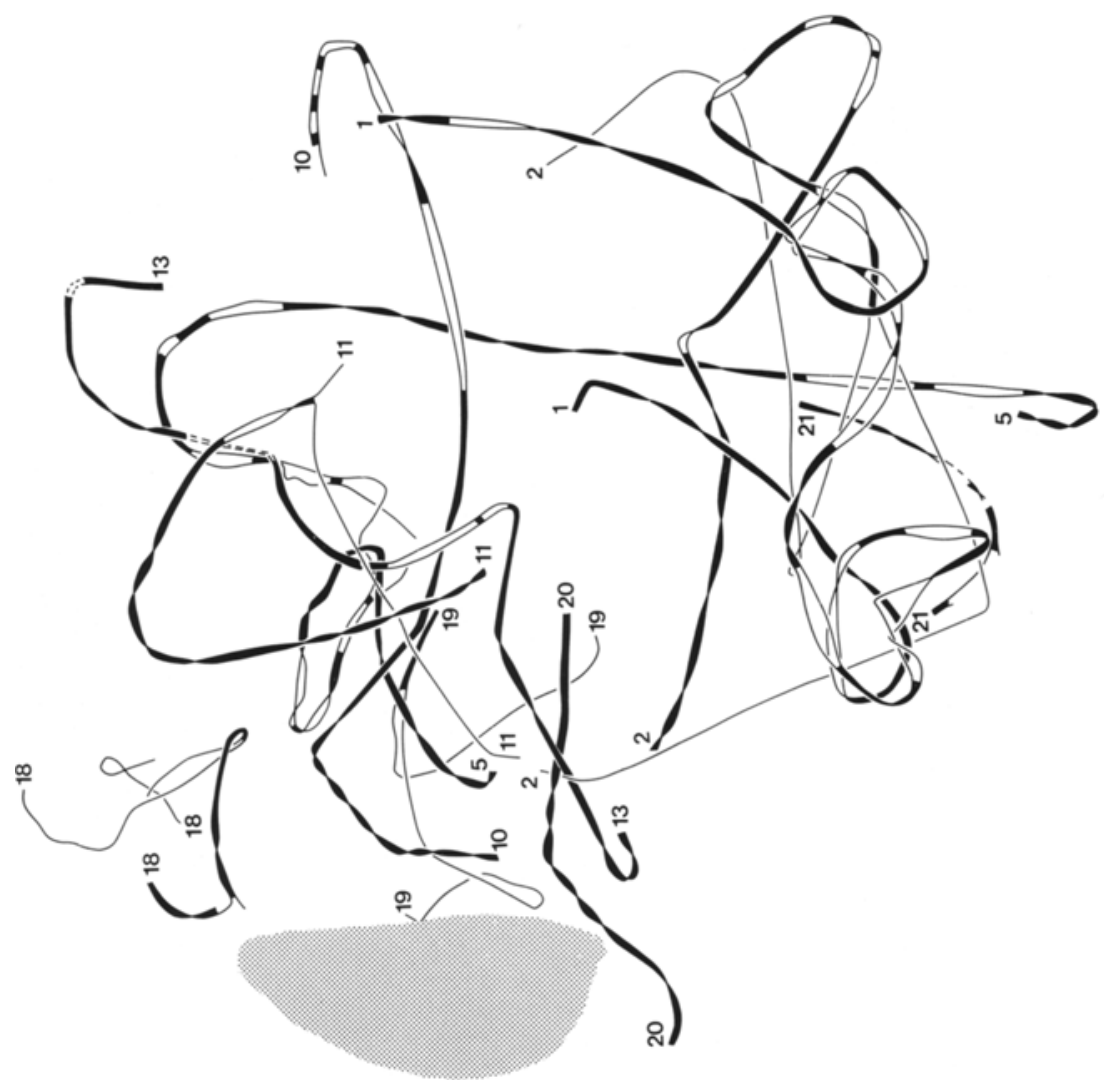

ס 
P.B.Holm: Synapsis in monosomic 5B wheat

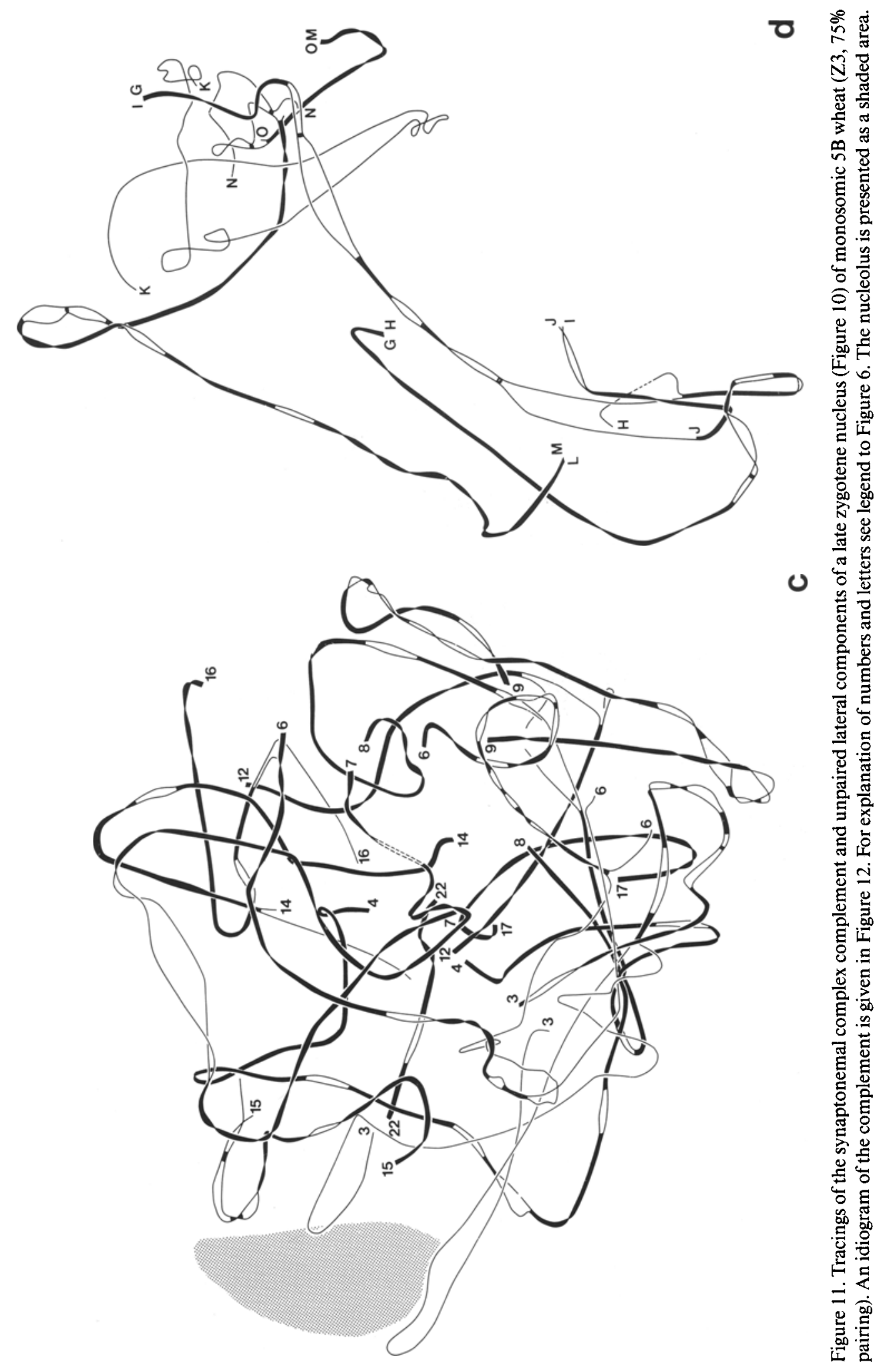




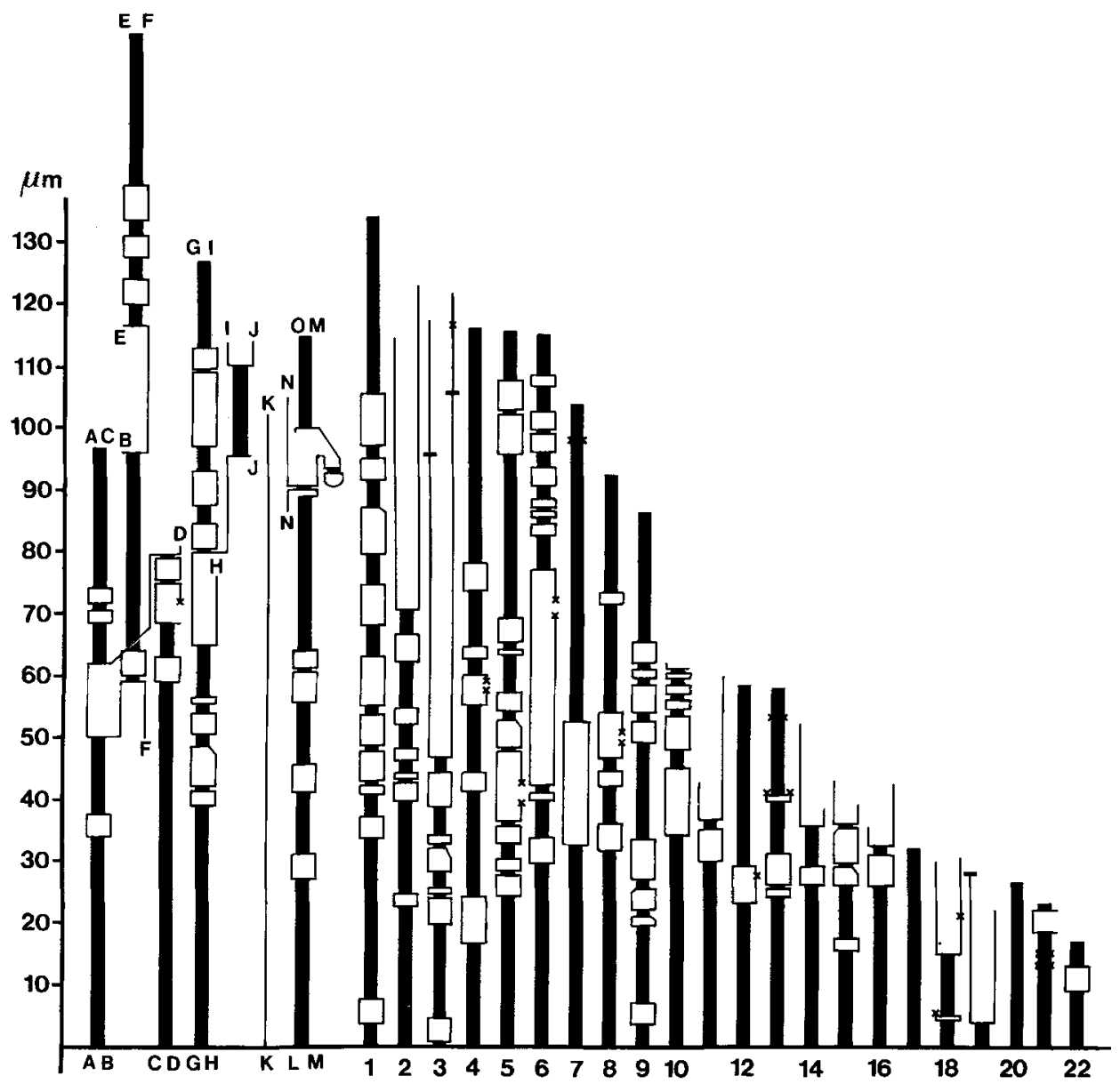

Figure 12. An idiogram of a spread late zygotene nucleus (Z3, 75\% pairing) of monosomic $5 B$ wheat. Tracings of the complement are given in Figure 11. For explanation of symbols see legend to Figure 7.

pairs $\mathrm{GH}, \mathrm{IJ}$ and $\mathrm{KM}$ (Figure 7), have probably been involved in multiple associations even though they appear as bivalent-like structures.

The quantitative data for the three early-mid zygotene nuclei are given in Table I. The mean number of lateral components or lateral component fragments involved in multiple associations amounts to 15 per nucleus of which four are engaged in switch back associations of type II.

In the previous study of euploid wheat (3), quantitative data were presented for a single nucleus with a pairing percentage below $50 \%$. Therefore, additional nuclei of euploid wheat were spread and four nuclei ranging in the degree of synapsis from $27 \%$ to $45 \%$ analyzed in detail (Table II, Figure 9). The mean degree of synapsis in the 5 nuclei was $39 \%$ which is almost the same as that achieved in the three nuclei from monosomic $5 B$ wheat $(42 \%)$. Also the mean lateral component length of the three genomes (half the total lateral component length) of the two genotypes was rather similar $(2342 \mu \mathrm{m}$ in monosomic 5B versus $1984 \mu \mathrm{m}$ in euploid wheat).

Major differences are, however, apparent when comparing the frequency of pairing partner exchange in the two genotypes. In mono- 
somic 5B wheat a mean of 15 lateral components or lateral component fragments per nucleus are involved in multiple associations, the corresponding number being 8 in euploid wheat. Furthermore, while nearly all multiple associations in euploid wheat are type II associations, most multiple associations in monosomic 5B wheat are types in which the lateral components only exchange pairing partners once resulting in long segments of synaptonemal complex on either side of the region of pairing partner exchange.

Apart from these differences pairing and synaptonemal complex formation appear to proceed basically in the same way in the two genotypes with an initiation of synapsis primarily from the telomeres and frequent interstitial initiation. The kinetics of pairing and synaptonemal complex formation have not been compared in a quantitative way in the two genotypes, but inspection of the idiograms did not reveal any obvious differences between them.

Differences between the two genotypes are, however, apparent with respect to the spreading behaviour of the nuclei. Nuclei from monosomic 5B wheat, and in particular the ones at early-mid zygotene, spread more easily than nuclei from euploid plants at the same stage. Although not analyzed in a quantitative way, one gets the impression that the congression of the telomeres to a small region of the nuclear envelope is more prominent and lasts longer in early-mid zygotene nuclei of euploid wheat than in nuclei from monosomic $5 \mathrm{~B}$ plants, where the bouquet configuration appears to be less compact and to disappear at an earlier stage of synapsis than in euploid wheat.

\subsubsection{Mid-late zygotene}

The analysis of the mid-late zygotene stage in monosomic $5 \mathrm{~B}$ wheat comprised complete tracings of six nuclei ranging in the degree of synapsis from $70 \%$ to $91 \%$ with a mean of $79 \%$ (Table III). Figure 10 is a montage of micrographs of a spread mid-late zygotene nucleus (Z3) with $75 \%$ synapsis. Tracings of the complement are shown in Figure 11 and an idiogram in Figure 12.

Multiple associations arising by pairing part- ner exchange between different combinations of lateral components were seen in all traced midlate zygotene nuclei. On the average the nuclei had ten lateral component or lateral component fragments involved in multiple associations (Table III). None of the associations were of type II.

As is the case at the early-mid zygotene stage, neither of the chromosomes can be identified. The nuclei contain, however, long unpaired lateral components (e.g. lateral component $K$ in Figures 11 and 12) which in most cases folds back and synapses with itself(Figures 12, 13 and 14 and univalent lateral components in nuclei $\mathrm{Z} 2$ and $\mathrm{Z4}$ in Figure 15). In some cases it synapses with itself at one end only, while the other end has formed a synaptonemal complex with another lateral component (nucleus Z6 in Figure 15). It is considered likely that this partially unpaired lateral component is chromosome 5B. As discussed in section 4.3 it is conceivable that the lateral components which have synapsed with the presumptive chromosome 5B in fact are the other members of the homoeologue group 5, i.e. chromosomes $5 \mathrm{~A}$ and 5D.

A comparison between the data obtained for mid-late zygotene nuclei of monosomic 5B wheat and those from euploid wheat (Table III) reveals that the complement lengths are basically the same in the two genotypes. However, as at early to mid zygotene, the number of lateral components involved in multiple associations is about two times higher in monosomic $5 \mathrm{~B}$ wheat than in the euploid.

\subsubsection{Pachytene}

Seventeen spread pachytene nuclei were traced in the present study (Table IV). Some of the nuclei appeared to be at the pachytenediplotene transition as judged by a high frequency of discontinuities of in particular the unpaired lateral components. The distinguishing characteristics between zygotene and pachytene are in monosomic 5B wheat as in euploid wheat an almost complete synapsis (Table IV), a change in the shape of the spread nuclei from circular to elongated-lobed (Figure 16) and a reduction in complement length by about onethird. 


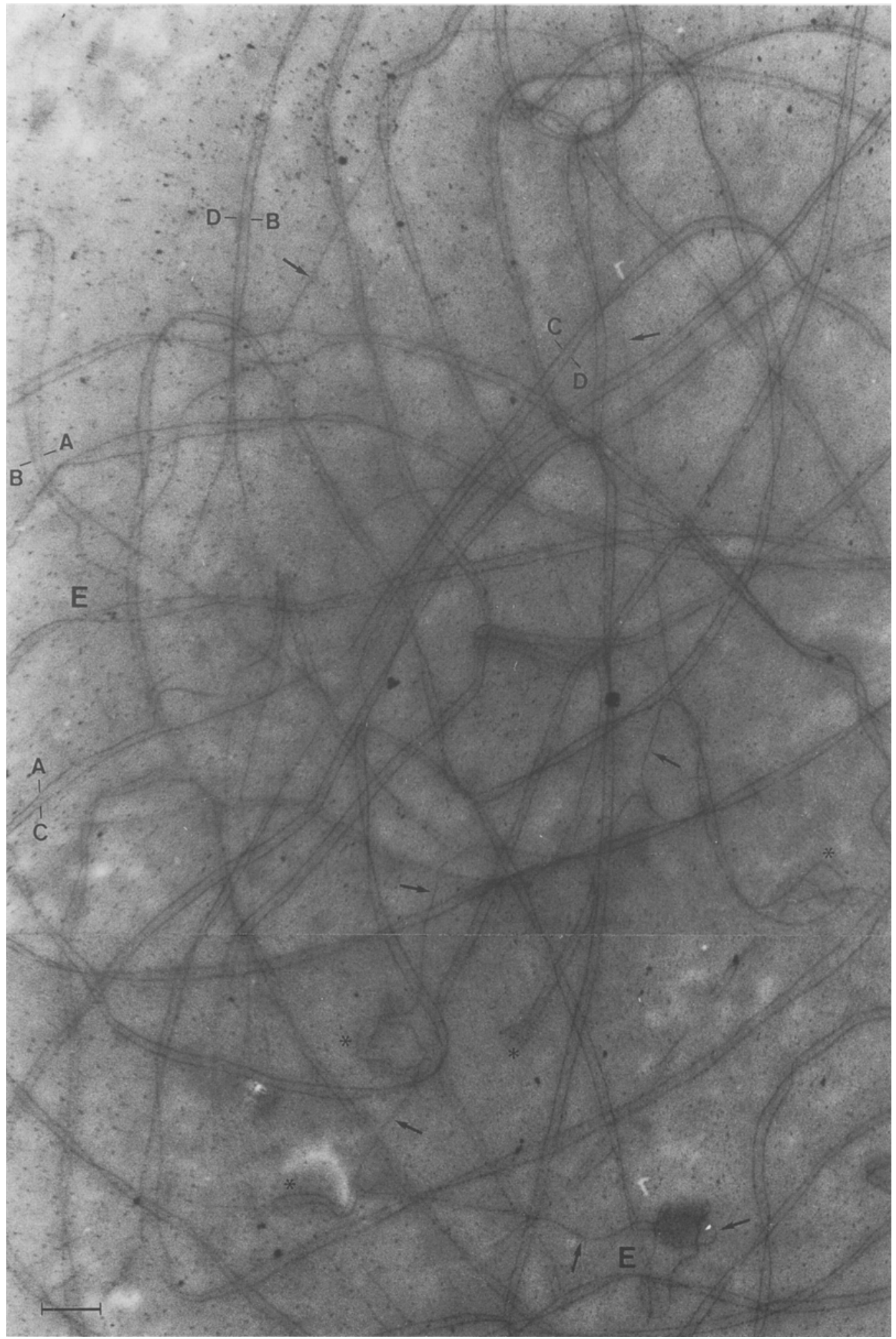


Figure 13. A montage of micrographs from a spread late zygotene nucleus of monosomic 5B wheat (Z4, 91\% pairing). The micrographs show a long single lateral component ( $E$, denoted by arrows) which in four regions has folded back and paired with itself (marked by asterisks). In addition, a multiple association involving four lateral components, marked by letters A to D, is apparent. A tracing of the five lateral components is given in Figure 14. $(\mathrm{Bar}=10 \mu \mathrm{m})$

Multiple associations of lateral components are at this stage as frequent as at mid to late zygotene (mean of 9 lateral components or lateral component fragments per nucleus involved in multiple associations at pachytene versus a mean of 10 at mid-late zygotene). Examples of multiple associations are shown in the tracings of the nucleus designated P17 (Figure 17), in the idiogram of the complement of this nucleus in Figure 18 in the micrographs in Figures 19 and 20 and in the idiogram of the multiple associations found in seven of the pachytene nuclei (Figure 21). In contrast to the situation at mid-late zygotene, long unpaired lateral components, i.e. the presumptive univalent chromosome 5B, are seldom seen, being replaced by foldback paired hairpin structures (e.g. in P7 and P10, Figure 21). In nearly all nuclei, however, the presumptive chromosome 5B has engaged in pairing and synaptonemal complex formation with other lateral components resulting in the formation of heteromorphic associations of two lateral components and multiple associations. As at the previous stage it is considered likely that chromosome $5 \mathrm{~B}$ preferentially has paired with the members of homoeologue group 5. The complement length in the spread pachytene nuclei is basically the same in monosomic 5B and euploid plants amounting to a mean of $1322 \mu \mathrm{m}$ in the former and 1474 $\mu \mathrm{m}$ in the latter (Table IV). In both genotypes the pachytene length is only about two-thirds of the length at mid zygotene.

A minor difference is observed in the mean degree of synapsis achieved in the two genotypes. As in euploid wheat the pachytene nuclei showing a high degree of synapsis were preferentially micrographed and the results suggest that synaptonemal complex formation is slightly more complete in the euploid plants (mean of $97 \%$ synapsis) than in the monosomic 5B plants (mean of $90 \%$ synapsis).
In contrast to this high degree of similarity between the two genotypes distinct differences are evident when comparing the frequency of multiple associations at this stage. Pachytene nuclei of monosomic 5B wheat contain a mean of 9 lateral components or lateral component fragments per nucleus in multiple associations in contrast to a mean of 0.6 in the euploid (Table IV).

\subsubsection{Early diplotene}

The early diplotene stage is, as in euploid wheat, distinguished from the pachytene stage

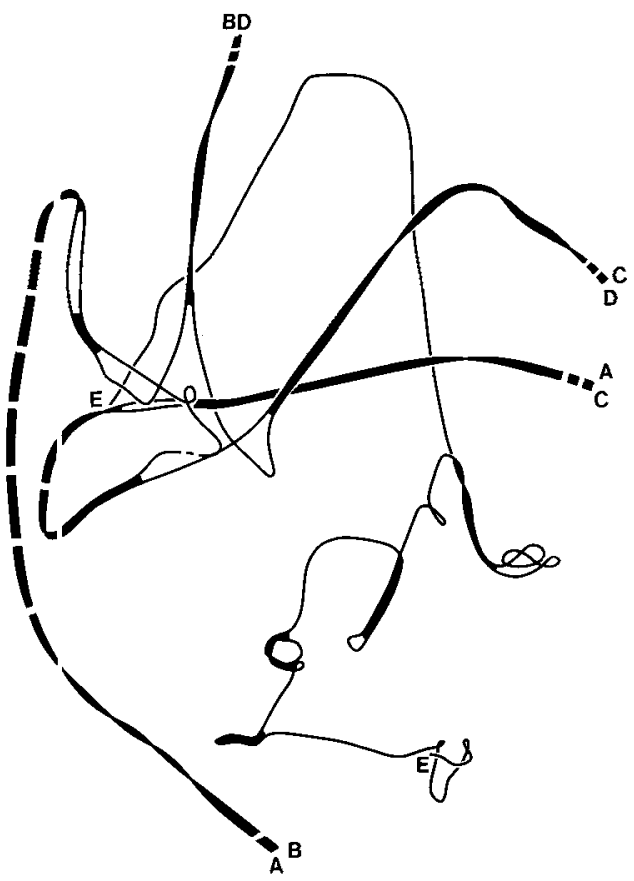

Figure 14. A tracing of the long unpaired lateral component and the association involving four lateral components shown in Figure 13. 


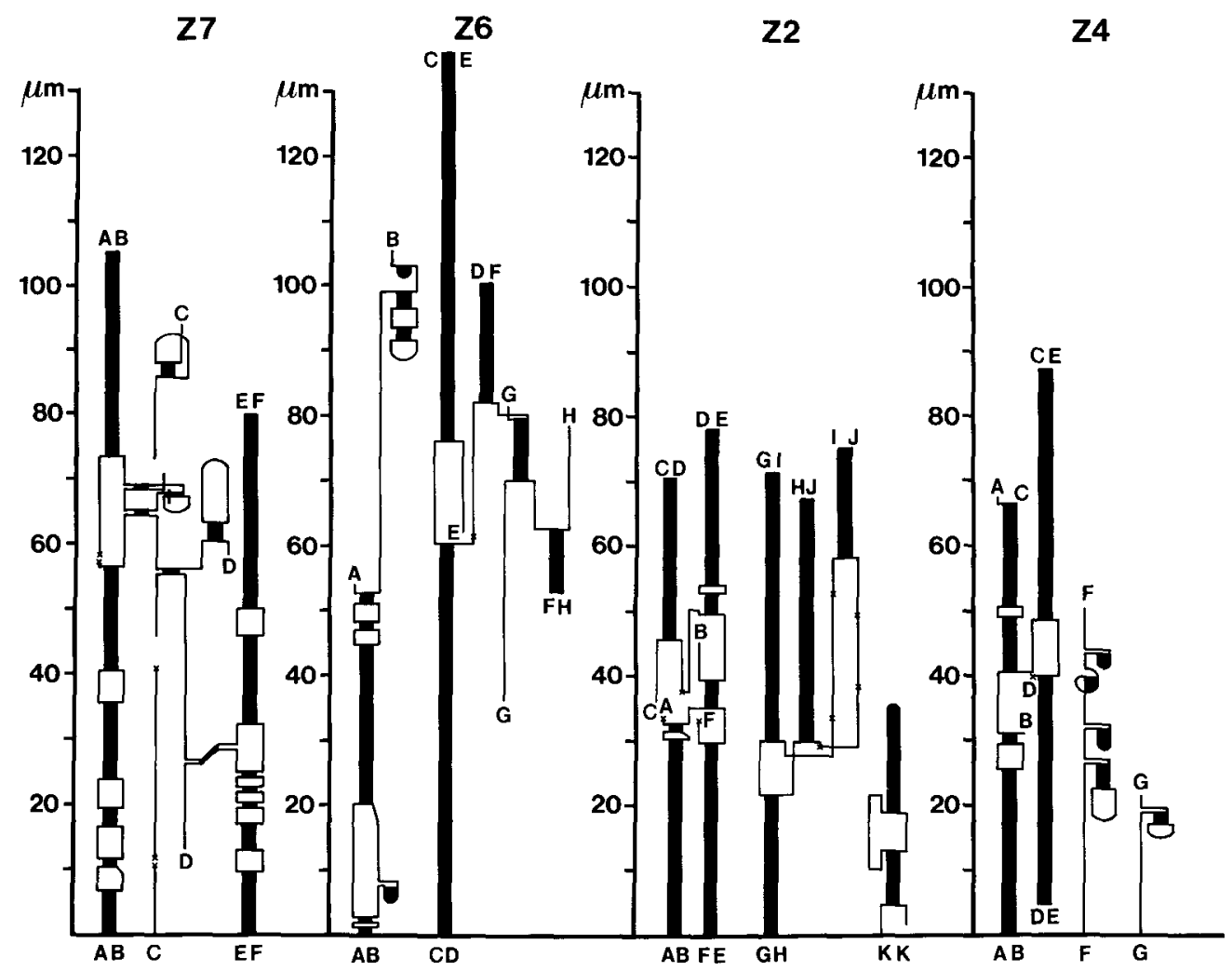

Figure 15. Idiograms of multiple associations of lateral components found in four mid-late zygotene nuclei. For explanation of symbols see legend to Figure 7.

by the initiation of lateral component degradation and by more diffuse chromatin, which in the silver-stained spreads result in less electron scattering background of the nuclei than at the preceding stages. As noted in the preceding section several of the spread nuclei, assigned to the pachytene stage, appeared rather to be at a transition stage between pachytene and diplotene, and likewise a few of the nuclei classified as early diploid nuclei might as well be assigned to a pachytene-diplotene transition stage. The nucleus shown in Figure 22, designated ED3 thus appears to be at the transition stage. Nuclei at later stages of diplotene were not included in this study, since lateral component degradation in these nuclei was too advanced to permit a quantitative evaluation of the number of lateral components in multiple associations. Such information was in fact only obtainable from two of the four early diploid nuclei analyzed. In both nuclei (Figure 23) the number of lateral components or lateral component fragments involved in multiple associations was the same as the mean number found at pachytene, i.e. about 10 per nucleus (Table V).

\section{DISCUSSION}

\subsection{General aspects}

A detailed description and discussion of the kinetics of chromosome pairing and synaptonemal complex formation as well as other ultrastructural characteristics of synapsis such as interlocking and interlocking resolution have been given previously for euploid wheat (3). Likewise, the general discussion of the effect of chromosome 5B is given elsewhere (6). The present discussion will therefore be restricted to 
Table IV. Degree of pairing, lateral component (LC) length of one complement $(1 / 2 \mathrm{LC}$ length) and the number of lateral components or lateral component fragments involved in multiple associations in seventeen pachytene nuclei of wheat microsporocytes monosomic for chromosome 5B. The number of multiple associations in parenthesis are switch back associations of type II (see section 3.2.2 for explanation). s.d., standard deviation. The data for euploid (2) are included for comparison.

\begin{tabular}{lllrl}
\hline $\begin{array}{l}\text { Nucleus } \\
\text { number }\end{array}$ & \% pairing & $\begin{array}{l}1 / 2 \text { LC length } \\
\mu \mathrm{m}\end{array}$ & \multicolumn{2}{c}{$\begin{array}{l}\text { Number of LC's in } \\
\text { multiple associations }\end{array}$} \\
\hline 1 & 91 & 1106 & 9 & $(0)$ \\
2 & 89 & 1244 & 15 & $(0)$ \\
3 & 87 & 1207 & 12 & $(6)$ \\
4 & 85 & 1187 & 13 & $(6)$ \\
5 & 76 & 1220 & 9 & $(0)$ \\
6 & 92 & 1177 & 13 & $(0)$ \\
7 & 94 & 1323 & 7 & $(0)$ \\
8 & 86 & 1188 & 8 & $(0)$ \\
9 & 94 & 1124 & 12 & $(4)$ \\
10 & 96 & 1181 & 7 & $(0)$ \\
11 & 86 & 1167 & 7 & $(0)$ \\
12 & 92 & 1482 & 6 & $(0)$ \\
13 & 91 & 1889 & 5 & $(0)$ \\
14 & 88 & 1527 & 9 & $(0)$ \\
15 & 98 & 1248 & 3 & $(0)$ \\
16 & 90 & 1533 & 7 & $(0)$ \\
17 & 96 & 1669 & 7 & $(0)$ \\
\hline Mean \pm s.d. & $90 \pm 5$ & $1322 \pm 220$ & $9 \pm 3(1)$ \\
\hline Euploid wheat & & & & \\
Pachytene & $97 \pm 7$ & $1474 \pm 214$ & 0.6 & $(0.3)$ \\
\hline
\end{tabular}

a summary of the results and a brief discussion of the differences encountered in meiosis between euploid wheat and monosomic $5 \mathrm{~B}$ wheat.

\subsection{Initiation of synapsis}

The present study has revealed that in monosomic $5 \mathrm{~B}$ wheat as well as in euploid wheat bouquet formation and initiation of synapsis is accompanied by an apparently unspecific alignment between short segments of lateral components. It has not been attempted to estimate if there are differences in these early alignment steps between monosomic 5B and euploid wheat. Apart from these alignments between a large number of different chromosomes, there is no indication for any large scale alignment of pairs or groups of chromosomes. Shortly thereafter when synaptonemal complex formation is in progress, most of the regions of alignment have been resolved and chromosome pairing and synaptonemal complex formation proceed primarily from the telomeres but also interstitially.

The local alignment between the many different combinations of lateral components observed at the leptotene to zygotene transition may be interpreted as transient events of mutual tests for homology between different combinations of lateral components. However, as evidenced by the relative scarcity of pairing partner exchanges at early zygotene, most of the local alignment events will not result in synaptonemal complex formation. As the telomeres already are bound to a limited area of the nuclear envelope their movements are restricted to a two dimensional space. On the other hand, the interstitial segments can move in three dimensions and an alignment sufficiently stable to trigger synaptonemal complex formation may 
P.B.Holm: Synapsis in monosomic 5B wheat

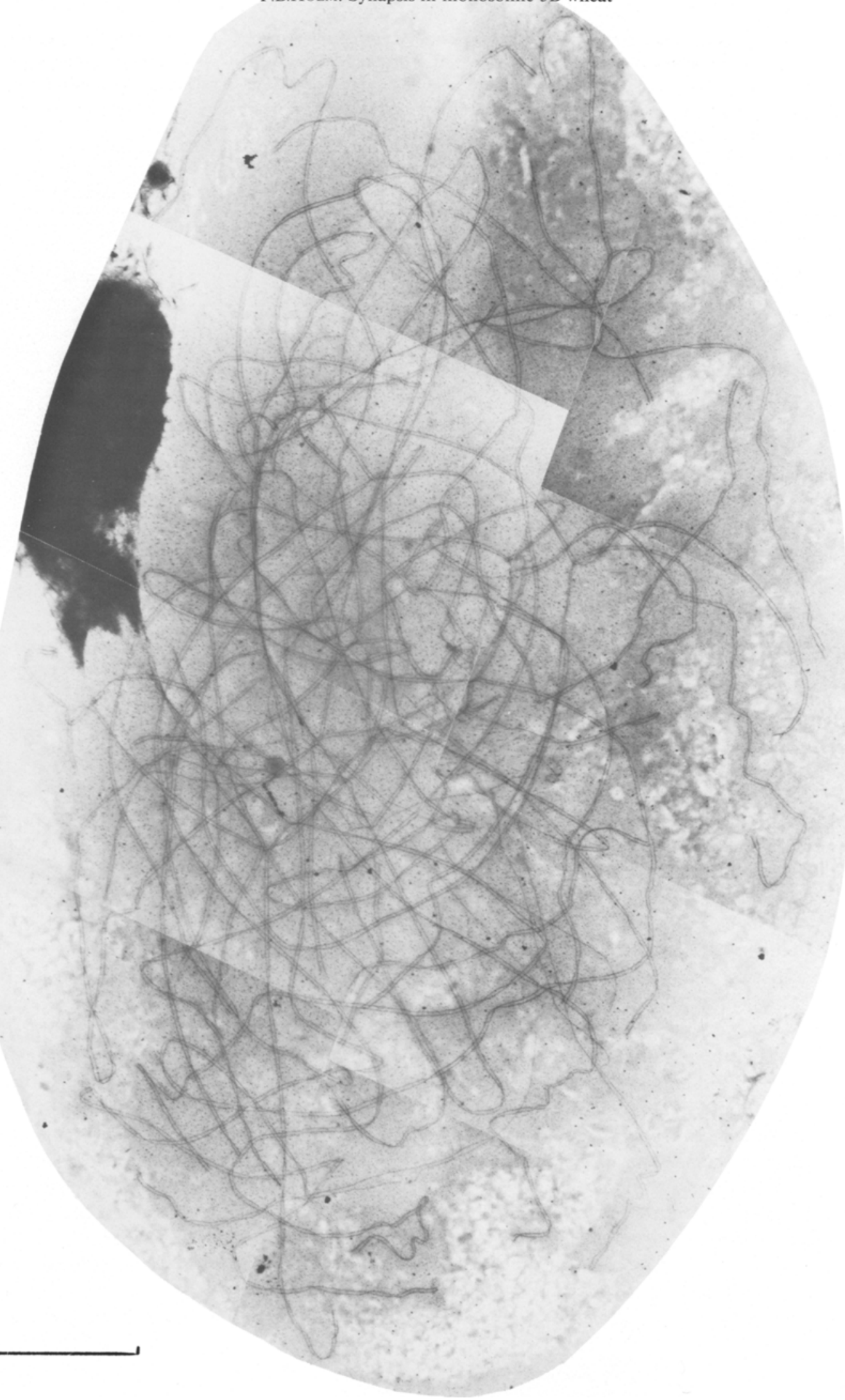


Figure 16. A montage of micrographs of a pachytene nucleus (P17,96\% pairing) of monosomic $5 \mathrm{~B}$ wheat. A tracing of the entire complement of this nucleus is shown in Figure 17 and an idiogram in Figure 18. $(\mathrm{Bar}=10 \mu \mathrm{m})$

therefore more easily be established in the telomeric regions.

\subsection{Multiple associations}

Throughout zygotene the number of lateral components in multiple associations is two times hlgher in monosomic 5B wheat than in euploid wheat. This may reflect a dosage effect of monosomy for chromosome $5 \mathrm{~B}$ on the stringency of chromosome pairing and synaptonemal complex formation. At least a part of the increase in pairing partner exchange may, however, also be attributed to the mere presence of a univalent chromosome. It is considered likely that univalency for chromosome $5 \mathrm{~B}$ results in a pairing partner competition among the five chromosomes of homoeologue group 5 in which the univalent chromosome 5B - because of partial homology - can compete with chromosomes $5 \mathrm{~A}$ and $5 \mathrm{D}$ for a partner. Some of the multiple associations observed are in fact indicative of pentavalent formation. A simple test of this hypothesis would be to count the number of lateral components in multiple associations at zygotene in a wheat strain monosomic for a chromosome not assumed to have any influence on the regulation of the disomic inheritance of allohexaploid wheat.

It is a general observation that pairing and synaptonemal complex formation at early stages are highly homologue specific while at later stages of zygotene synaptonemal complex formation is less or not specific at all $(9,14)$. The presence of long unpaired segments of the presumptive chromosome 5B at early zygotene and the more complete synapsis of this chromosome at late zygotene-pachytene conform with this notion. It should be realized though that the individual chromosomes cannot be identified and therefore the long univalent lateral components, which often fold back on themselves, just as well might be other chromosomes such as $5 \mathrm{~A}$ or 5D. This might explain the occasional presence of additional univalents at metaphase I.
The ring shape often attained by these chromosomes as well as by chromosome 5B could be attributed to fold back pairing and subsequent crossing over and chiasma formation between the distal parts of the two arms. A more likely explanation, however, is that the association between the distal parts of the arm is a nonchiasmatic one, resulting from incomplete elimination of the synaptonemal complex in the hairpin structure during diplotene or it reflects an association of the chromatin of a foldback paired univalent. Such nonchiasmatic associations between univalents, assumed to result from previous synapsis into pairs or multiple associations are also frequent at metaphase $I$ in wheat $\times$ rye hybrids (12).

\subsection{Correction of pairing}

In monosomic $5 \mathrm{~B}$ there is the same number of lateral components in multiple associations at mid-late zygotene as at pachytene in contrast to the situation in euploid wheat, where multiple associations are almost completely eliminated. This indicates differences in the process of synaptic correction. As noted previously (3) there is an excellent correlation between meiotic stage and anther length in euploid wheat. The same correspondance between anther length and stage is found in monosomic $5 \mathrm{~B}$ wheat and the duration of the stages should therefore be very similar in the two genotypes. Possibly the pachytene stage is slightly longer in monosomic 5B wheat than in euploid wheat as pachytene nuclei are relatively frequent in spreads of monosomic 5B wheat. As can be judged from the data in Tables I and III-V, the number of lateral components involved in multiple associations only decreases from early-mid zygotene to mid-late zygotene, where switch back associations of type II are primarily affected, while the numbers of lateral components in multiple associations from mid-late zygotene to pachytene (9-10 per nucleus) and perhaps even at early diplotene are basically the same. It is possible 


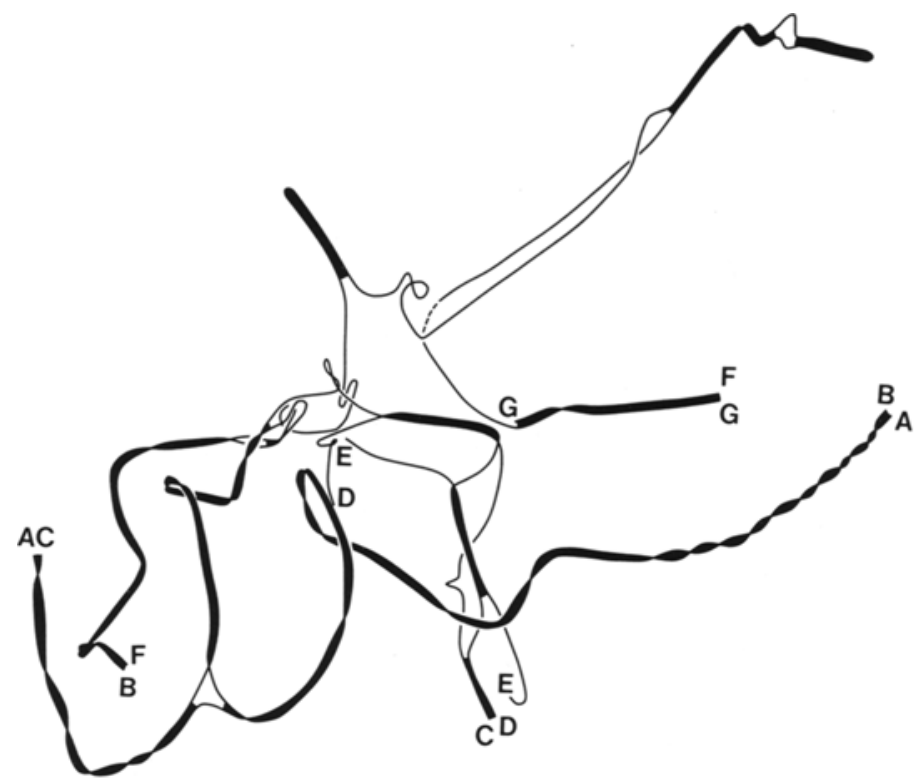

a

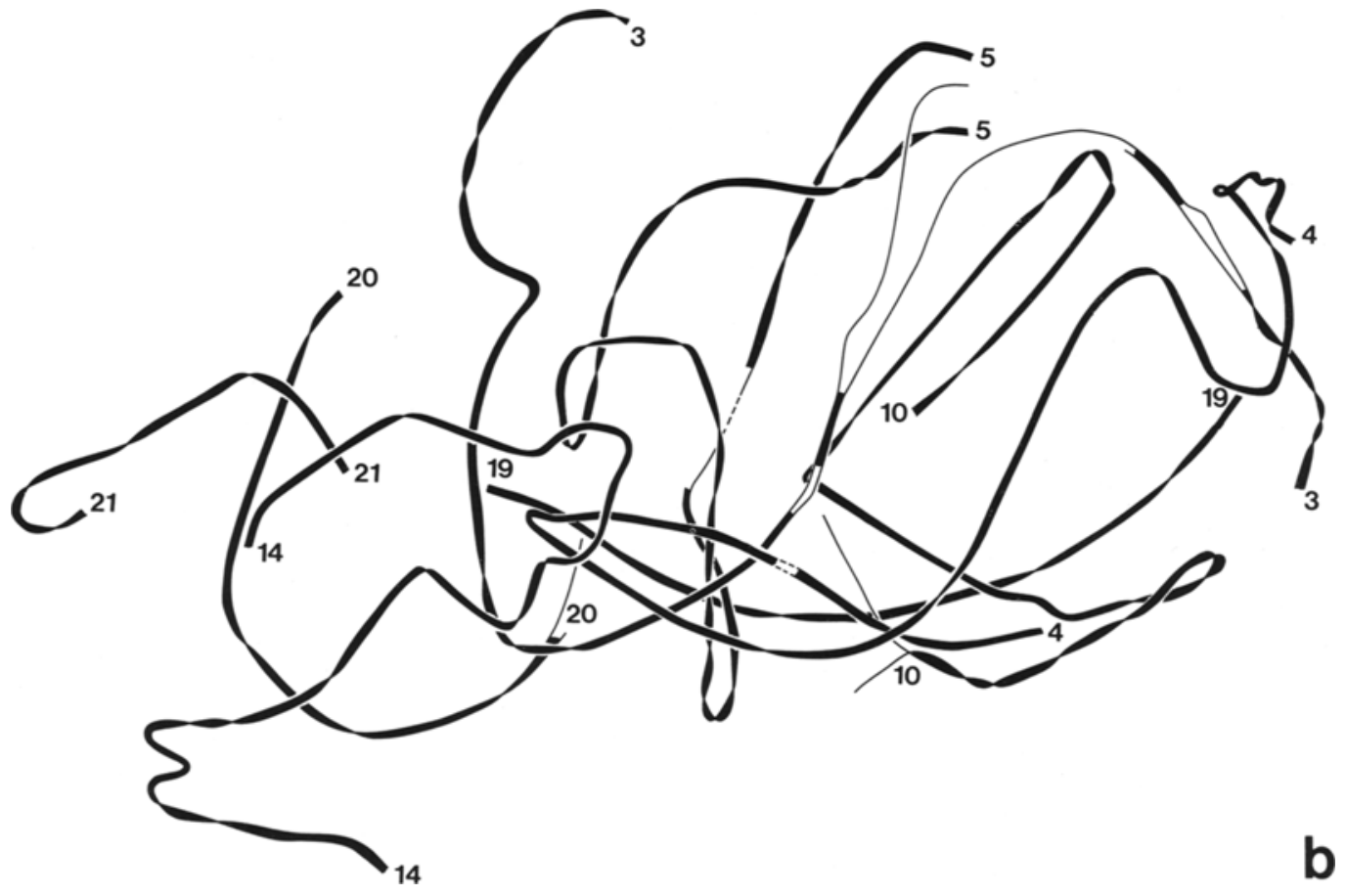

that some correction takes place whereby the region for pairing partner exchange shifts towards the telomeres, but as can be seen in the idiograms in Figure 23 the pairing partner exchanges are still a considerable distance from the telomeres, implying that at most only a limited movement of the site of pairing partner exchange has occurred.

As evidenced by the absence of multivalents at metaphase $\mathrm{I}$, crossing over does not occur in the 


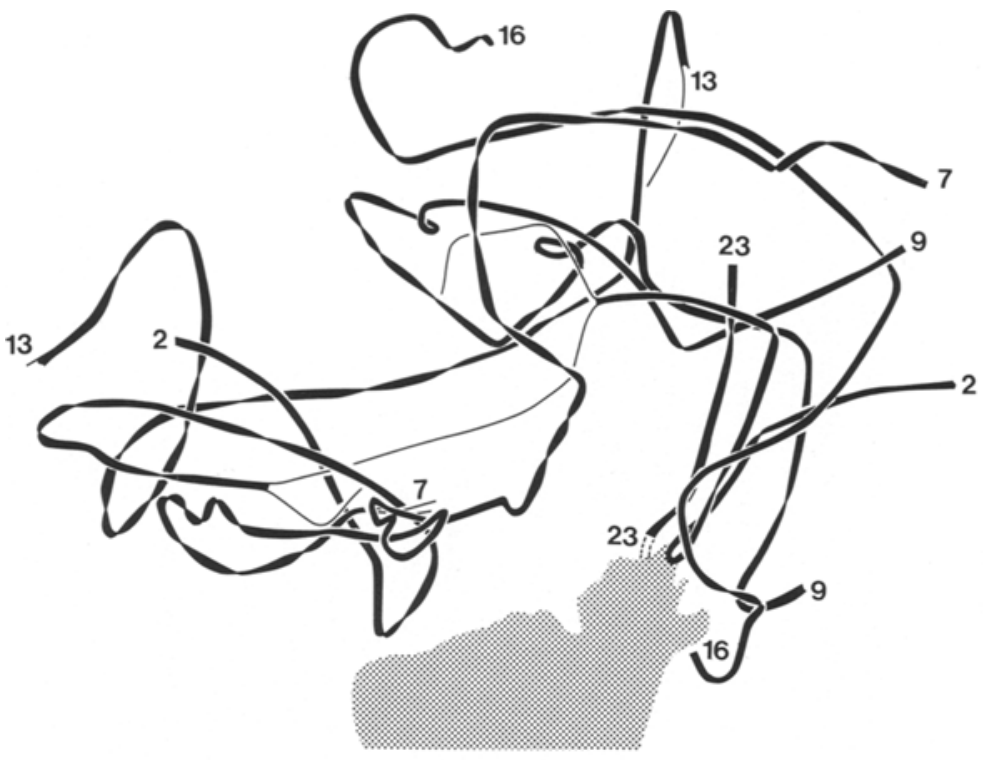

C

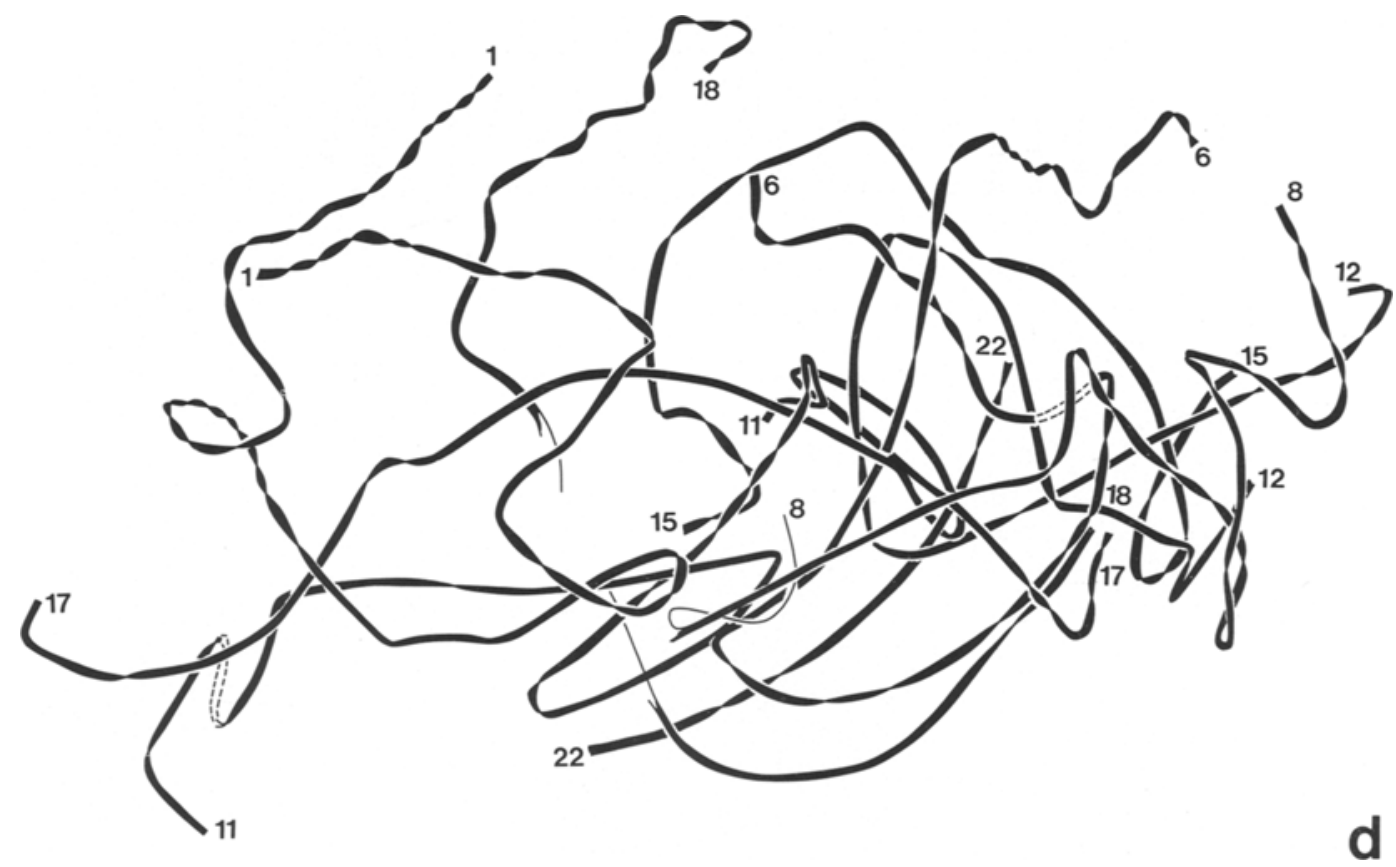

Figure 17. Tracings of the entire complement of a spread pachytene nucleus (P17, 96\% pairing) of monosomic 5B wheat. The nucleolus is marked by a shaded area. For explanation of numbers and letters see legend to Figure 6 .

retained multiple associations in monosomic $5 \mathrm{~B}$ wheat, and the lack of correction can therefore not be attributed to an inhibition of correction by crossing over. Possibly, the presumptive pen- tavalent association may be less prone to correction due to the lack of a homologous partner to chromosome 5B. This explanation is, however, not considered likely either. In triploid oocytes 


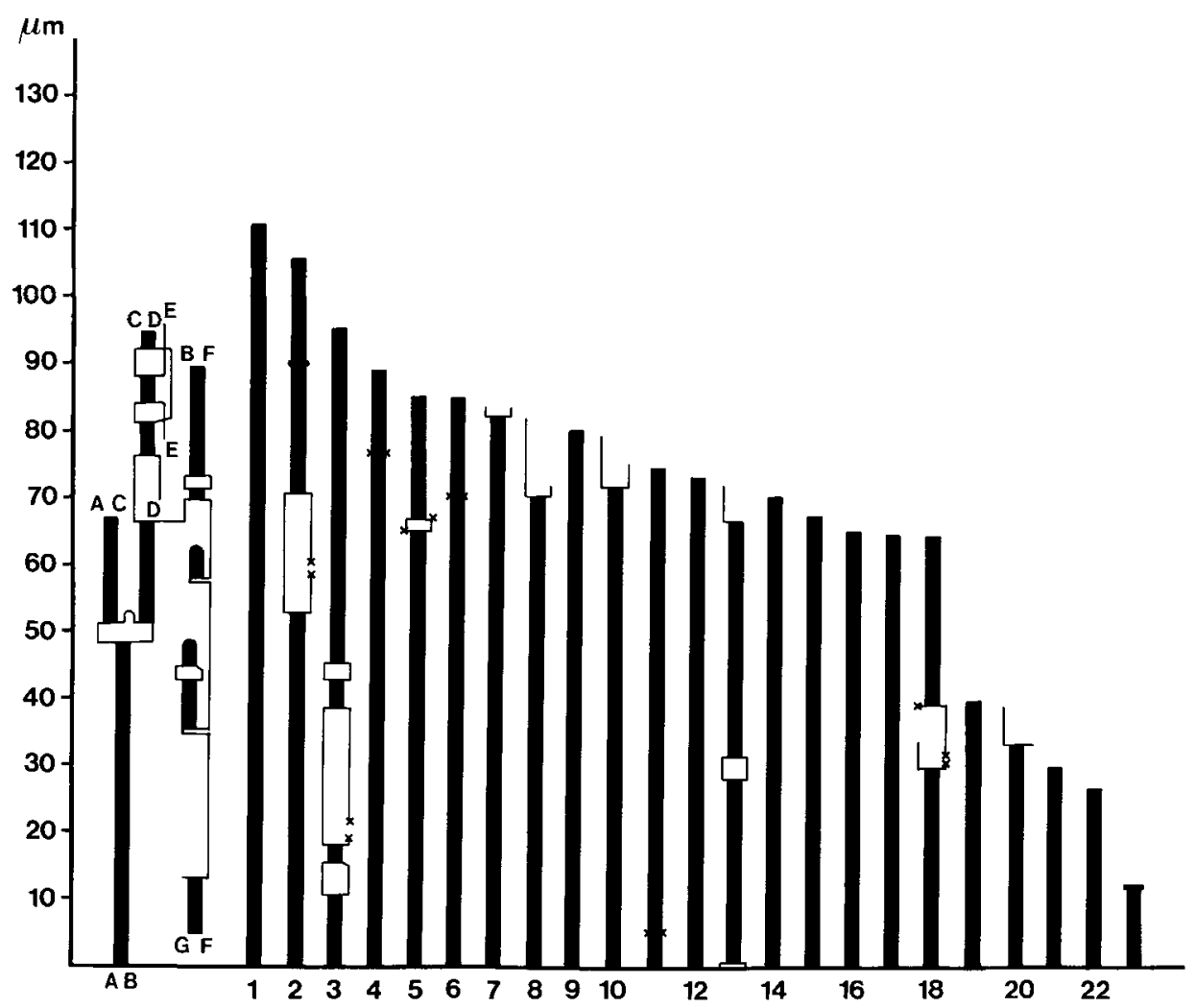

Figure 18. An idiogram of the entire complement of synaptonemal complexes and lateral components in a spread nucleus of monosomic 5B wheat. The nucleolus organizing regions are marked with bars. For explanation of symbols see legend to Figure 7.

of the female silkworm Bombyx mori, where crossing over does not occur, several trivalents are formed during zygotene, but are subsequently corrected into bivalents and univalents at pachytene. The univalents then engage in various nonhomologous associations with other uniyalents or undergo foldback pairing (9). The most likely explanation which can account for these differences between monosomic $5 \mathrm{~B}$ and euploid wheat is that correction stops at an earlier stage in the former genotype. Analysis of pachytene nuclei selected for having a high degree of synapsis in the two genotypes thus revealed a mean of $90 \%$ synapsis in monosomic 5B and $97 \%$ synapsis in euploid wheat. As the ongoing process of synapsis appears to be the driving force in correction (6), the lack of correction in monosomic $5 \mathrm{~B}$ wheat may be attributed to a less complete synapsis in this genotype.

\subsection{Suppression of crossing over between homoeologues}

In monosomic 5B wheat there is no crossing over and chiasma formation between homoeologues as judged by the genetic stability of this line and the absence of multivalents at metaphase $I$, in spite of the presence of multiple associations at zygotene and pachytene. Among the associations are the presumptive pentavalents involving the five homoeologues of group 5 and it is considered likely that several of the other multiple associations also result from pairing and synaptonemal complex formation between homoeologues. An analogous situation is described for pentaploid wheat (genomic constitution $2 \times \mathrm{A}, 2 \times \mathrm{B}, 1 \times \mathrm{D}$ ), obtained by crossing $T$. aestivum with $T$. durum, where only eight percent of the metaphase I complements contain a trivalent not withstanding monosomy for 

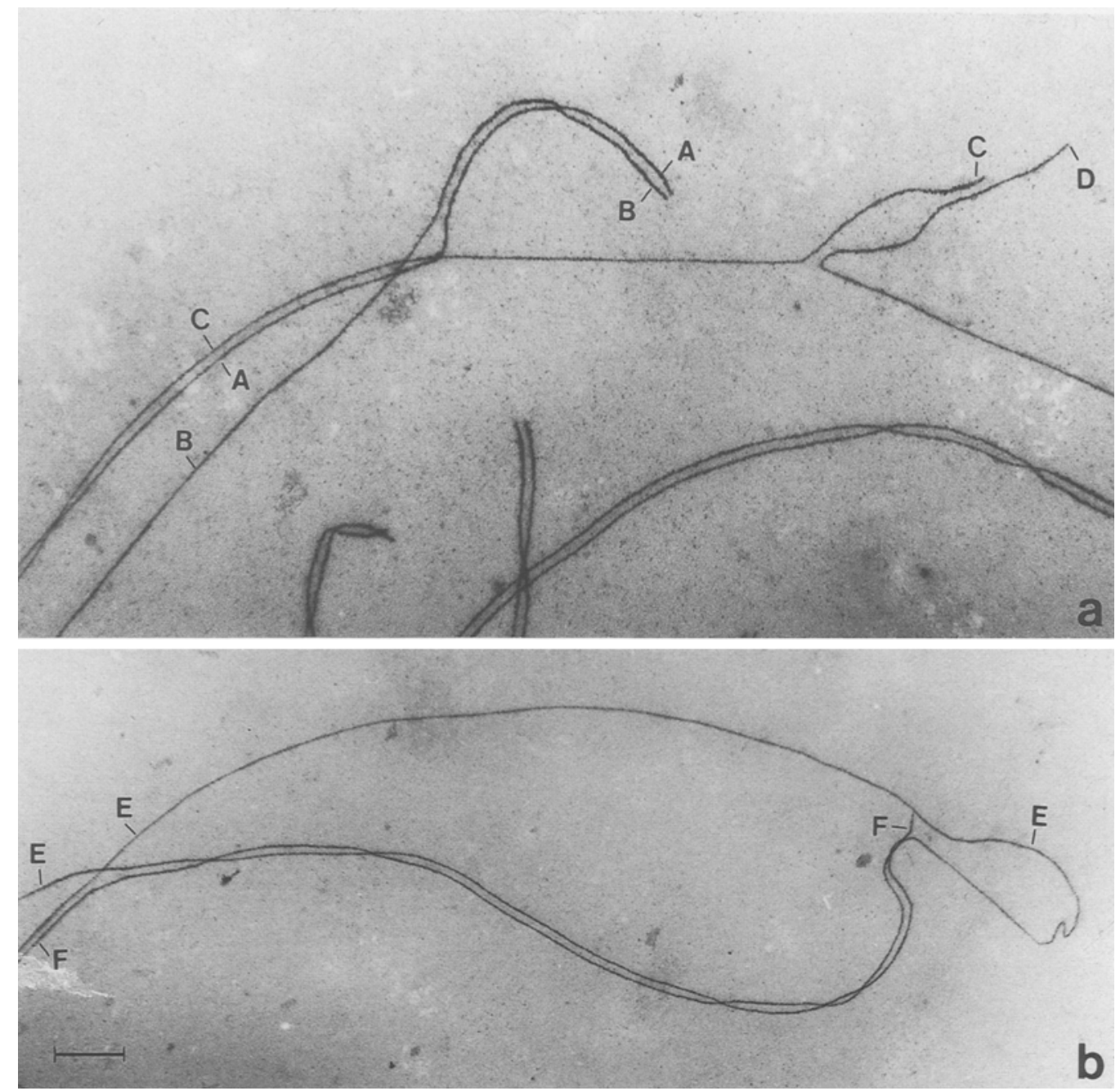

Figure 19. Two micrographs showing examples of pairing partner exchanges in a spread pachytene nucleus (P5, $76 \%$ pairing) of monosomic $5 \mathrm{~B}$ wheat. In Figure $19 \mathrm{a}$ is shown a shift of pairing partner close to the telomeres, and in Figure $19 \mathrm{~b}$ a pairing configuration characteristic for an inversion. (Bar $=1 \mu \mathrm{m})$

all the seven chromosomes of the D genome (8).

The present study has thus disclosed that monosomy for chromosome $5 \mathrm{~B}$ results in a twofold increase in the number of lateral components in multiple associations compared to euploid wheat. Most multiple associations of chromosomes are retained through pachytene. The presence of one copy of chromosome 5B is sufficient to suppress crossing over between homoeologues.

\section{ACKNOWLEDGEMENTS}

I am indebted to Professor DITER VON Wettstein, M. Sc. Xingzhi Wang and Dr. SØREN W. RASMUSSEN for discussion and review of the manuscript. The expert technical assistance of Bibi Stampe ANDERSEN, JeAn SAGE, NinA RASMUSSEN and ANN-Sofi STEINHOLTZ is gratefully acknowledged. The Royal Danish Academy of Sciences and Letters are thanked for having provided me with a Niels Bohr 


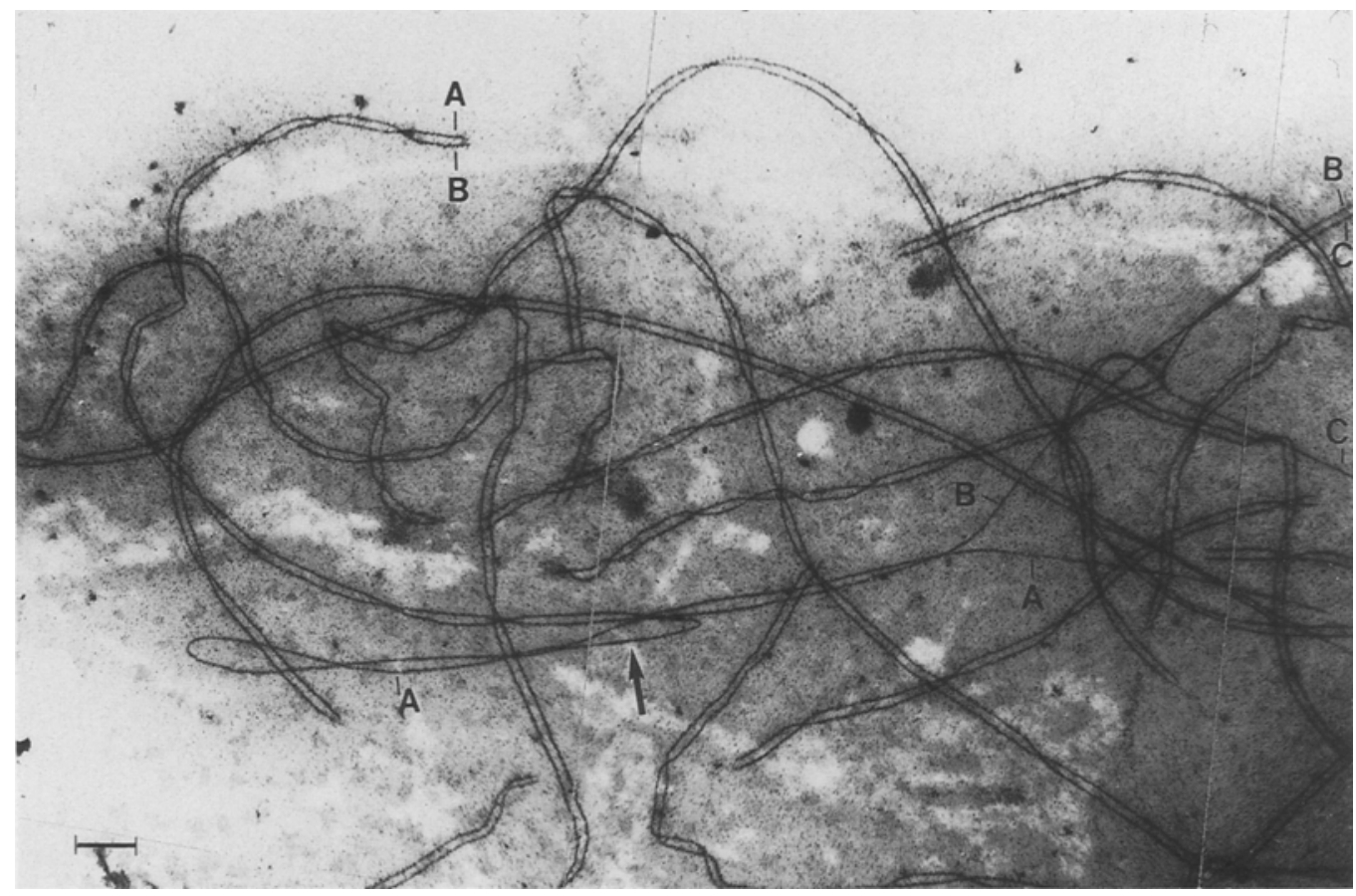

Figure 20. A montage of micrographs showing a pairing partner exchange in a spread pachytene nucleus $(\mathrm{PI}, 91 \%$ pairing) of monosomic 5B wheat. The lateral components involved in the muitiple association are denoted by capital letters. The lateral component marked $\mathrm{A}$ is considerably longer than $\mathrm{B}$ and a large unpaired loop has formed (arrow). $(\mathrm{Bar}=10 \mu \mathrm{m})$

Table V. Degree of pairing, lateral component (LC) length of one complement $(1 / 2, L C$ length) and the number of lateral components or lateral component fragments involved in multiple associations in four early diplotene nuclei of wheat microsporocytes monosomic for chromosome $5 \mathrm{~B}$. The number of multiple associations in parenthesis are switch back associations of type II (see section 3.2.2 for explanation). ND, not determined. s.d., standard deviation.

\begin{tabular}{|c|c|c|c|}
\hline $\begin{array}{l}\text { Nucleus } \\
\text { number }\end{array}$ & $\%$ pairing & $\begin{array}{l}1 / 2 \text { LC length } \\
\mu \mathrm{m}\end{array}$ & $\begin{array}{l}\text { Number of LC's in } \\
\text { multiple associations }\end{array}$ \\
\hline 1 & 86 & 1757 & $10 \quad(0)$ \\
\hline 2. & 85 & 1248 & $9 \quad(0)$ \\
\hline 3 & 76 & 1170 & ND \\
\hline 4 & 88 & 1240 & ND \\
\hline Mean \pm s.d. & $84 \pm 5$ & $1353 \pm 271$ & $10 \quad(0)$ \\
\hline
\end{tabular}

Figure 21. Idiograms of the multiple associations found in seven spread pachytene nuclei of monosomic $5 \mathrm{~B}$ wheat. The double arrow in the idiogram of pachytene nucleus $P 10$ points to two alternative interpretations of the pairing of several lateral component fragments. The lateral components denoted I and J in P6 are included among the multiple associations as they reveal a long region of inverted pairing. 
B.HoLm: Synapsis in monosomic $5 \mathrm{~B}$ wheat

P3

P1

P6

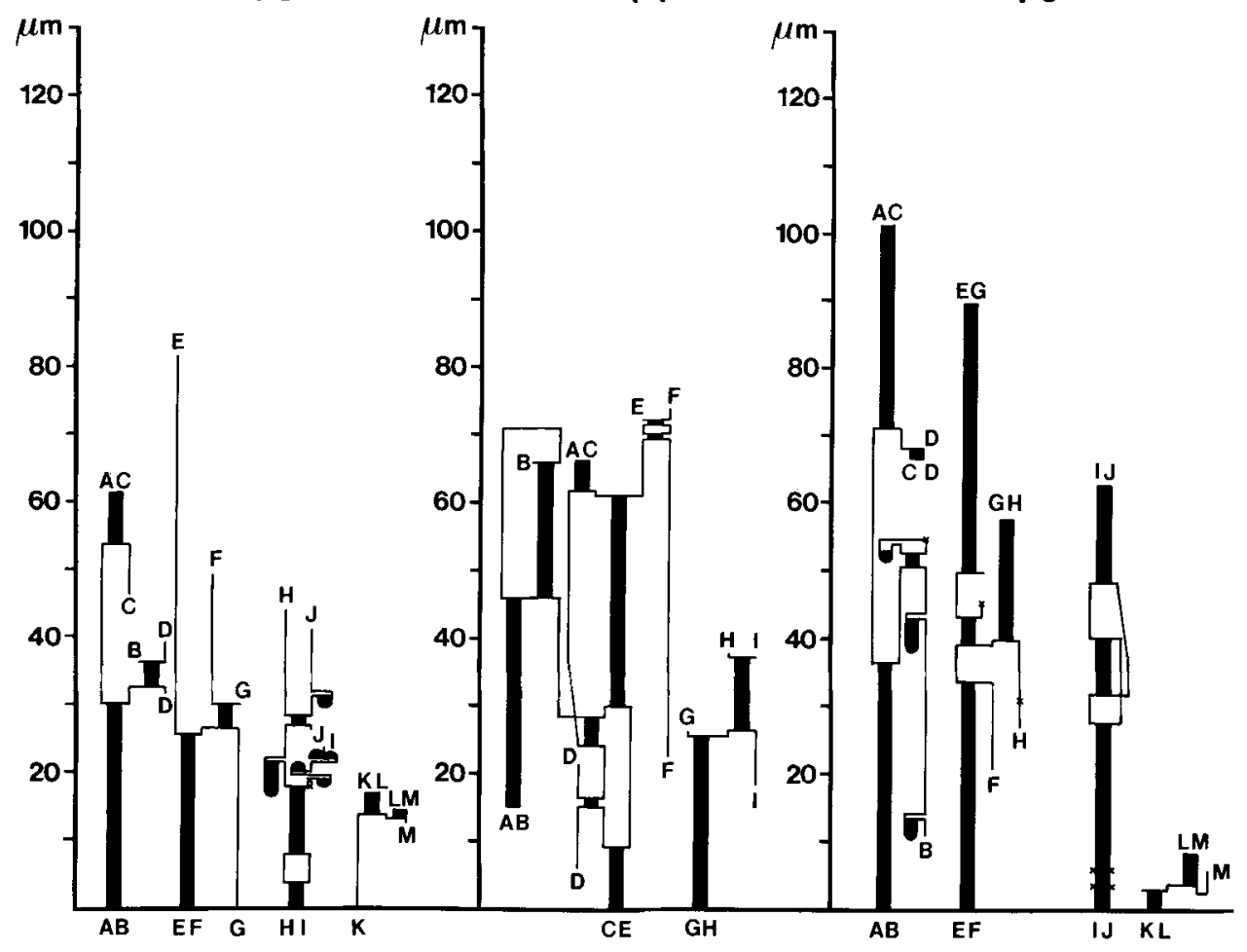

a

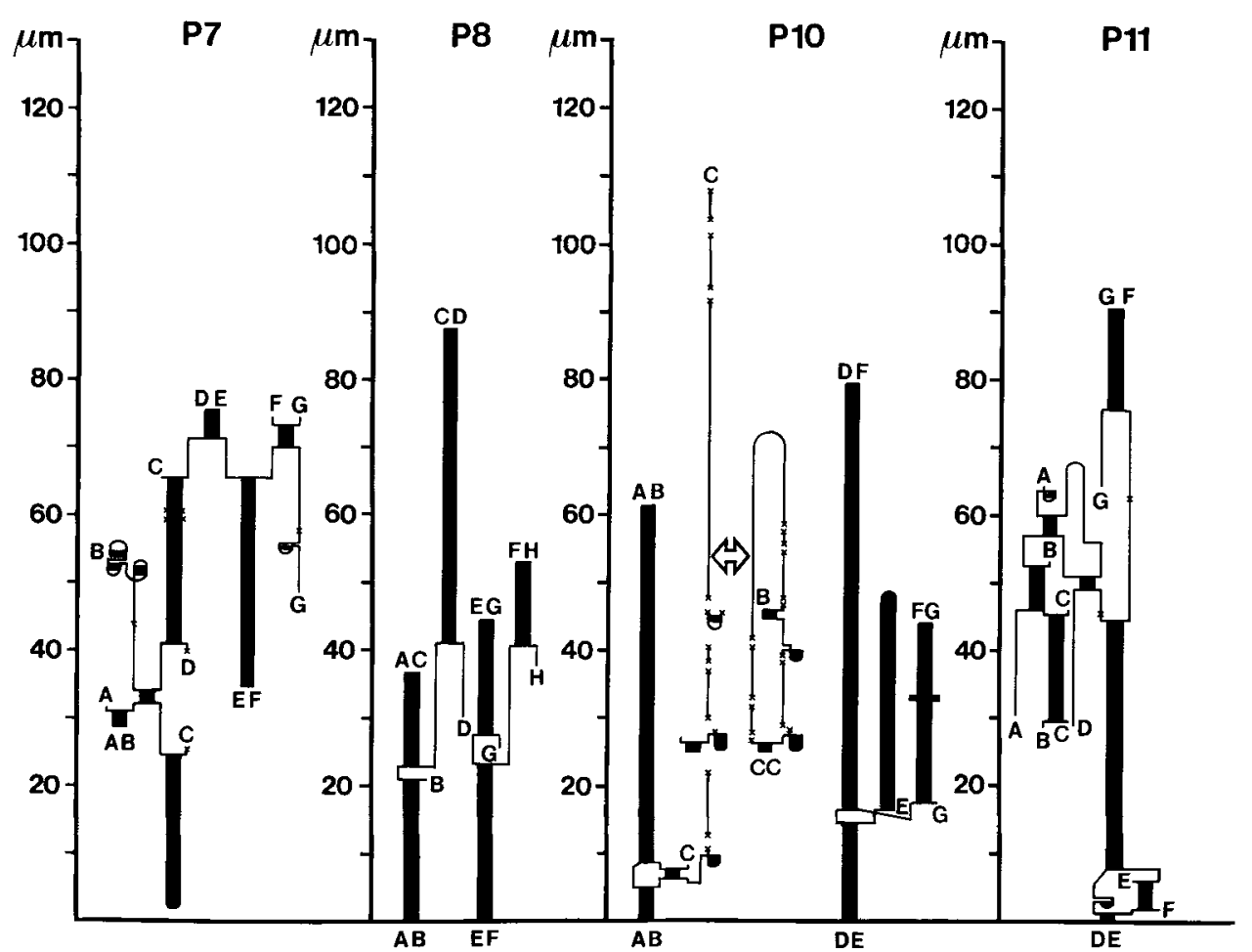


P.B.HoLM: Synapsis in monosomic 5B wheat

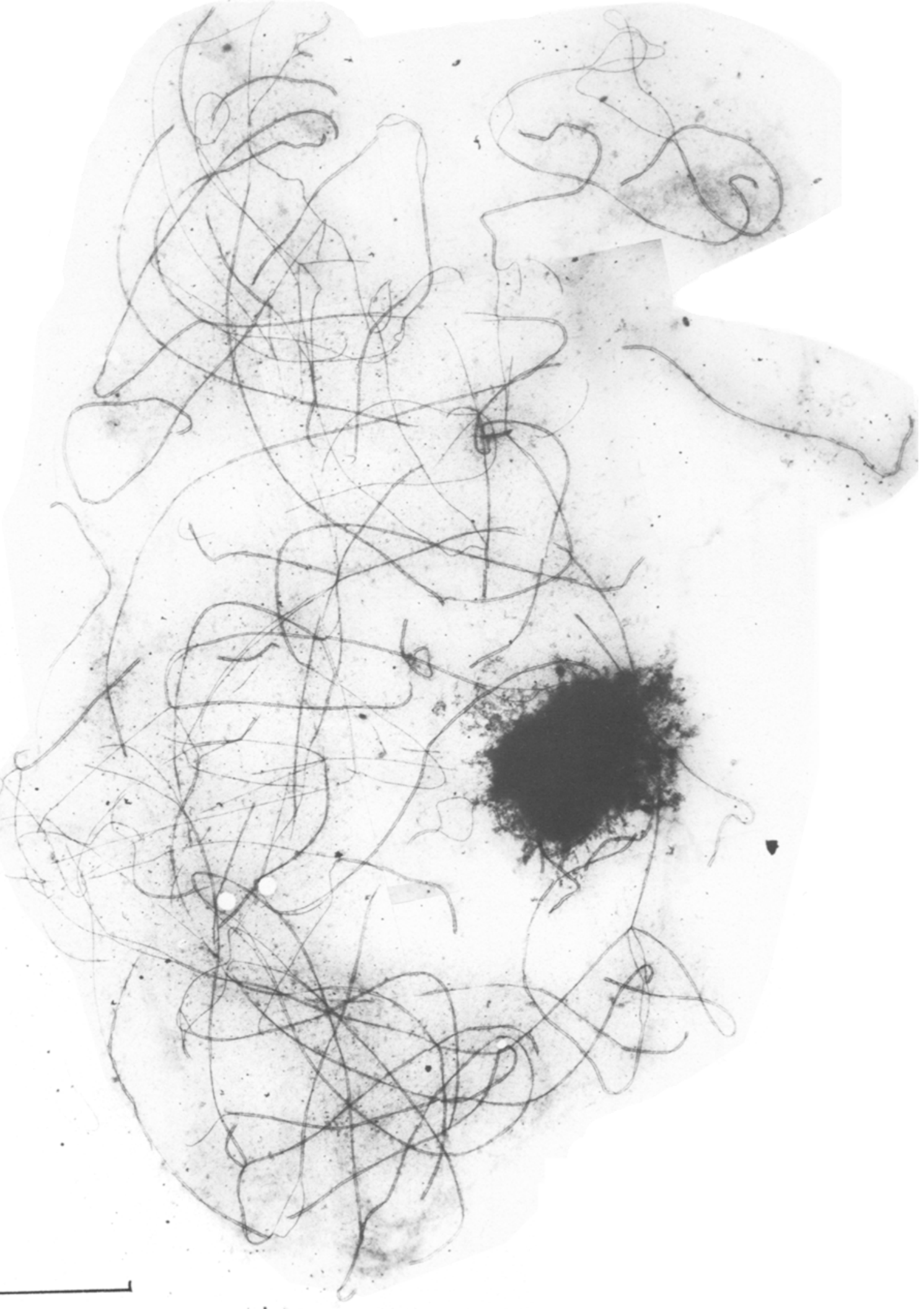


Figure 22. A montage of micrographs of a spread early diplotene nucleus (ED3) of monosomic 5B wheat. (Bar = $10 \mu \mathrm{m})$

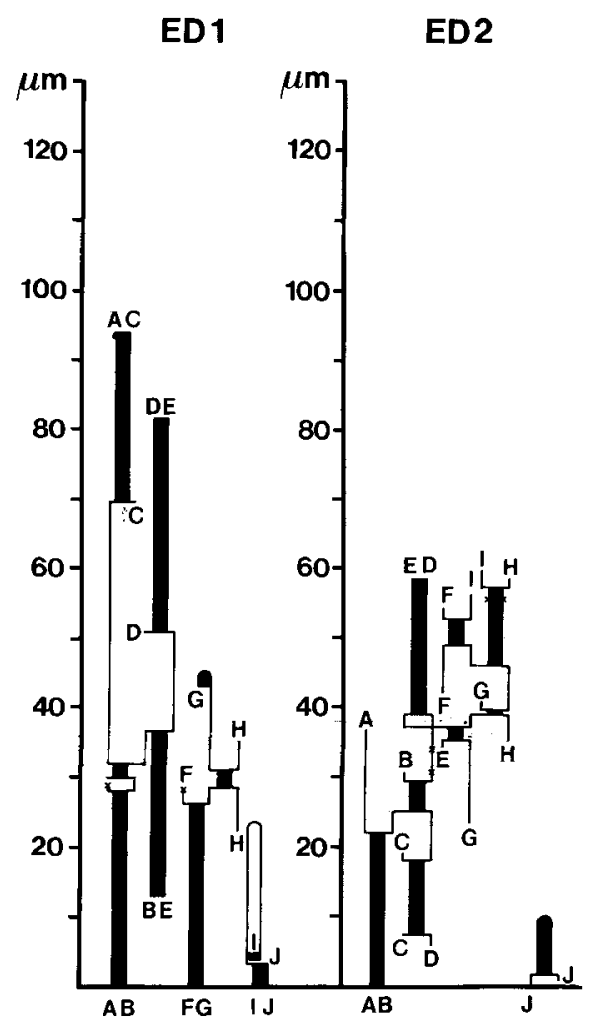

Figure 23. Idiograms of multiple associations found in two spread early diplotene nuclei of monosomic 5B wheat. For explanation of symbols see legend to Figure 7 .

Scholarship. The work was also supported by grant BI6-E-168-DK from the Commission of the European Communities.

\section{REFERENCES}

1. ENDO, T.R. \& B.S. GiLL: Somatic karyotype, heterochromatin distribution, and nature of chromosome differentiation in common wheat, Triticum aestivum L. em Thell. Chromosoma (Berl). 89, 361-369 (1984)

2. Новоцтн, P.: Chromosome pairing in allohexaploid wheat var. Chinese Spring. Transformation of multivalents into bivalents, a mechanism for exclusively bivalent formation. Carlsberg Res. Commun. 46, 129-173 (1981)
3. Holm, P.B.: Chromosome pairing and chiasma formation in allohexaploid wheat, Triticum aestivum analyzed by spreading of meiotic nuclei. Carlsberg Res. Commun. 51, 239-294 (1986)

4. HoLm, P.B.: Chromosome pairing and synaptonemal complex formation in hexaploid wheat, nullisomic for chromosome 5B. Carlsberg Res. Commun. 53, 91-110 (1988)

5. HoLm, P.B.: Chromosome pairing and synaptonemal complex formation in hexaploid wheat, monoisosomic and diisosomic for the long arm of chromosome 5B. Carlsberg Res. Commun. 53, 111-133 (1988)

6. HoLm, P.B.\& X. WANG: The effect of chromosome $5 B$ on synapsis and chiasma formation in wheat, Triticum aestivum cv. Chinese Spring. Carlsberg Res. Commun. 53, 191-208 (1988)

7. Jenkins, G.: Chromosome pairing in Triticum aestivum cv. Chinese Spring. Carlsberg Res. Commun. 48, 255-283 (1983)

8. Mello-Sampayo, T.: Homoeologous chromosome pairing in pentaploid hybrids of wheat. Proc. 3rd International Wheat Genetics Symposium pp. 179-184 (1968)

9. RASMUSSEN,S.W.: Chromosome pairing in triploid females of Bombyx mori analyzed by three-dimensional reconstructions of synaptonemal complexes. Carlsberg Res. Commun. 42, 163-197 (1977)

10. SEARS, E.R.: The aneuploids of common wheat. Mo. Agric. Exp. Sta. Res. Bull. 572, 58pp (1954)

11. WANG, $X$ : Chromosome pairing analysis in haploid wheat by spreading of meiotic nuclei. Carlsberg Res. Commun. 53, 135-166 (1988)

12. WANG, X.\& P.B. Hol.M: Chromosome pairing and synaptonemal complex formation in wheat-rye hybrids. Carlsberg Res. Commun. 53, 167-190 (1988)

13. WANG, X.\& H. Hu: The chromosome constitution of plants derived from pollen of hexaploid triticale $x$ common wheat $F_{1}$ hybrids. Theor. Appl. Genet. 70, 92-96 (1985)

14. Wettstein, D. von, S.W. Rasmussen \& P.B. Holm: The synaptonemal complex in genetic segregation. Ann. Rev. Genet. 18, 331-431 (1984)

15. WISCHMANN, B.: Chromosome pairing and chiasma formation in wheat plants triisosomic for the long arm of chromosome 5B. Carlsberg Res. Commun. 51, 1-25 (1986)

Accepted by E. LuND. 